\title{
Developmental Origin of PreBötzinger Complex Respiratory Neurons
}

\author{
Paul A. Gray, ${ }^{1}$ John A. Hayes, ${ }^{2,3}$ Guang Y. Ling, ${ }^{1}$ Isabel Llona, ${ }^{4}$ Srinivasan Tupal, ${ }^{1}$ Maria Cristina D. Picardo, ${ }^{3}$ \\ Sarah E. Ross, ${ }^{5}$ Tsutomu Hirata, ${ }^{6}$ Joshua G. Corbin, ${ }^{6}$ Jaime Eugenín, ${ }^{4}$ and Christopher A. Del Negro ${ }^{3}$ \\ ${ }^{1}$ Department of Anatomy and Neurobiology, Washington University School of Medicine, St. Louis, Missouri 63110, Departments of ${ }^{2}$ Biology and ${ }^{3}$ Applied \\ Science, College of William and Mary, Williamsburg, Virginia 23187, ${ }^{4}$ Laboratorio de Sistemas Neurales, Departamento Biología, Facultad de Química y \\ Biología, Universidad de Santiago de Chile, USACH, Santiago 9170022, Chile, ${ }^{5}$ Department of Neurobiology, Harvard Medical School, Boston, \\ Massachusetts 02139, and ' ${ }^{C}$ enter for Neuroscience Research, Children’s National Medical Center, Washington, DC 20010
}

A subset of preBötzinger Complex (preBötC) neurokinin 1 receptor (NK1R) and somatostatin peptide (SST)-expressing neurons are necessary for breathing in adult rats, in vivo. Their developmental origins and relationship to other preBötC glutamatergic neurons are unknown. Here we show, in mice, that the "core" of preBötC SST ${ }^{+} / \mathrm{NK} \mathrm{R}^{+} / \mathrm{SST} 2 \mathrm{a}$ receptor ${ }^{+}$(SST2aR) neurons, are derived from Dbx1-expressing progenitors. We also show that Dbx1-derived neurons heterogeneously coexpress NK1R and SST2aR within and beyond the borders of preBötC. More striking, we find that nearly all non-catecholaminergic glutamatergic neurons of the ventrolateral medulla (VLM) are also Dbx1 derived. PreBötC SST ${ }^{+}$neurons are born between E9.5 and E11.5 in the same proportion as non-SST-expressing neurons. Additionally, preBötC Dbxl neurons are respiratory modulated and show an early inspiratory phase of firing in rhythmically active

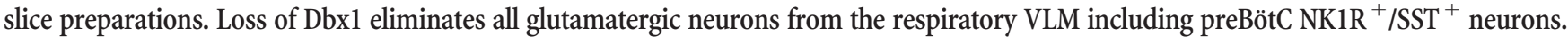
Dbx1 mutant mice do not express any spontaneous respiratory behaviors in vivo. Moreover, they do not generate rhythmic inspiratory activity in isolated en bloc preparations even after acidic or serotonergic stimulation. These data indicate that preBötC core neurons represent a subset of a larger, more heterogeneous population of VLM Dbx1-derived neurons. These data indicate that Dbx1-derived neurons are essential for the expression and, we hypothesize, are responsible for the generation of respiratory behavior both in vitro and in vivo.

\section{Introduction}

The necessity for the brainstem in the generation of breathing has been known for over a millennium, and the search for the regions most important for this behavior has been ongoing for nearly 200

\section{Received Aug. 2, 2010; revised Sept. 6, 2010; accepted Sept. 11, 2010.}

This work was supported by The College of William and Mary Science Education and Research Program funded by the Howard Hughes Medical Institute (J.A.H., C.A.D.N.), Fondo Nacional de Desarrollo Científico y Tecnológico Grants 1090375 and 7070065 (I.L., J.E.), Jane Coffin Childs and Dystonia Medical Research Foundation Awards (S.E.R.), National Institute of Neurological Disorders and Stroke (NINDS) Grant 1 F31 NS071860-01 (M.C.D.P.), NINDS Grant R21 NS070056-01 (C.A.D.N.), National Heart, Lung, and Blood Institute (NHLBI) Grant R01 HL104127-01 (C.A.D.N.), National Institute on Drug Abuse (NIDA) GrantDA020140 (T.H., J.G.C.), the Parker B. Francis and McDonnell Research Foundations (P.A.G.), and NHLBI Grant R01 HL089742 (G.L., S.T., P.A.G.). The content is solely the responsibility of the authors and does not necessarily represent the official views of the NHLBI, NINDS, NIDA, or National Institutes of Health. We thank Marcelo Otárola and George McMurray for technical assistance. We thank the following for advice, assistance, discussion, and biological resources that made this work possible: Tom Jessell for Dbx ${ }^{\mathrm{LacZ} /+}$ mice and Lmx1b antibody; Randy Johnson for Lmx1b ${ }^{\text {LacZ/+ }}$ mice; Josh Huang for Gad1-GFP mice; Frank Constantini for Rosa26-EYFP mice; Qiufu Ma for SIc6a5 and Gad1 in situ hybridization (ISH) probes; Martyn Goulding for Evx1 ISH probe, antibody, and Evx1 mutant mice; and Michael Greenberg for unpublished bHLHb5 data. P.A.G. conceived the study. J.A.H., M.C.D.P., and C.A.D.N. carried out intracellular recordings and statistical analysis. G.Y.L. sectioned brains and performed IHC and ISH. P.A.G. acquired, analyzed, and quantified anatomical data. S.T. performed in vivo video taping and analysis and in vitro mutant recordings. J.E. performed quantal slowing experiments. J.E. and I.L. performed and analyzed quantal slowing data. S.E.R., T.H., and J.G.C. provided unpublished resources, technical expertise, and edited the manuscript. J.A.H.. I.L., and S.T. edited the manuscript. C.A.D.N., J.E., and P.A.G. designed the experiments and wrote the manuscript.

Correspondence should be addressed to Paul A. Gray at the above address. E-mail: pgray@pcg.wustl.edu.

J. A. Hayes's present address: Institut de Neurobiologie Alfred Fessard, CNRS, 91190 Gif sur Yvette, France.

T. Hirata's present address: Ehime Proteo-Medicine Research Center, Ehime University, Toon, Ehime 791-0295, Japan.

D0I:10.1523/JNEUROSCI.4031-10.2010

Copyright $\odot 2010$ the authors $\quad 0270-6474 / 10 / 3014883-13 \$ 15.00 / 0$ years (Legallois, 1813; Feldman and Del Negro, 2006). A small region of the ventrolateral medulla (VLM), the preBötC, is hypothesized to be the site for respiratory rhythm generation (Smith et al., 1991). In the adult rat, a subset of glutamatergic neurons within this region coexpress the neurokinin 1 receptor (NK1R) and somatostatin (SST) peptide (Gray et al., 1999; Stornetta et al., 2003; Llona and Eugenín, 2005). The near-complete, targeted ablation of preBötC NK1R neurons over several days produces ataxic breathing during wakefulness, while the partial elimination of this population produces sleep-disordered breathing (Gray et al., 2001; McKay et al., 2005). The genetic silencing of preBötC neurons by expression of a Drosophila peptide receptor under the control of a fragment of the SST promoter induces persistent apnea (Tan et al., 2008). Together these data indicate an important role for a subset of preBötC neurons in breathing. What role glutamatergic neurons lacking SST or NK1R expression within the preBötC, as well as within the adjacent Bötzinger Complex (BötC) and rostral ventral respiratory group (rVRG), have in the generation of respiratory behavior is unknown (Feldman and Del Negro, 2006).

Transcription factor mutations that affect rhombomeric patterning and interneuron specification lead to respiratory instability and death, suggesting a strong genetic component to the formation of the respiratory central pattern generator (Blanchi et al., 2003; Rhee et al., 2004; Gray, 2008; Pagliardini et al., 2008; Rose et al., 2009; Thoby-Brisson et al., 2009; Caubit et al., 2010). No direct relationship, however, has been identified for any specific 
transcription factor and the formation of preBötC glutamatergic neurons. In contrast, within the VLM, discrete transcription factors give rise to respiratory glycinergic neurons (Lbx1), as well as retrotrapezoid nucleus and parafacial respiratory group (RTN/pFRG) glutamatergic neurons (Atoh1, Lbx1, Phox2b) (Pagliardini et al., 2008; Rose et al., 2009; Thoby-Brisson et al., 2009). Here we examined the role of the transcription factor Dbx1 in the genetic organization of the mammalian respiratory oscillator including preBötC neurons. Understanding the developmental and genetic organization of the neurons necessary for breathing may provide insight into the general principles of neural circuit formation as well as human breathing disorders.

\section{Materials and Methods}

Transgenic mice. We used Dbx $1^{\text {LacZ }}$ (Pierani et al., 2001), Dbx1 ${ }^{\text {CreERT2 }}$ (Hirata et al., 2009), Gad1 ${ }^{\text {GFP }}$ transgenic (Chattopadhyaya et al., 2004), Lmx1b ${ }^{\text {LacZ }}$ (Pressman et al., 2000), Rosa26-EYFP (Srinivas et al., 2001), Slc6a5 BAC transgenic (Gong et al., 2003), or wild-type mice. All mice were bred onto a mixed CD1/C56B6 or CF1 background. Experiments were done in accordance with the Institute for Laboratory Animal Research Guide for the Care and Use of Laboratory Animals. All experiments were approved by the Animal Studies Committee at Washington University School of Medicine, the Institutional Animal Care and Use Committee at the College of William and Mary, or the Bioethics Committee of the Universidad de Santiago de Chile.

Immunohistochemistry. Tissue sections were washed in PBS with $0.2 \%$ Triton X-100, blocked in 10\% heat-inactivated normal horse sera, incubated in antibody overnight at $4^{\circ} \mathrm{C}$, incubated in secondary antibody, and coverslipped in Vectashield.

Antibodies. Rat anti-bHLHb5 (1:1000, M. E. Greenberg, Harvard Medical School, Boston, MA), chicken anti- $\beta$-galactosidase ( $\beta$-gal) (1: 4000 , Abcam), rabbit anti- $\beta$-gal (1:1000, Covance), goat anti-FoxP2 (1: 2000, Abcam), chicken anti-green fluorescent protein (GFP) $(1: 1000$, Aves Labs), rabbit anti-GFP (1:2000, Invitrogen), goat anti-Lhx9 [1:1000, Santa Cruz Biotechnology (SCBT)], guinea pig anti-Lmx1b (1: 500, T. Jessell, Howard Hughes Medical Institute, Columbia University, New York, NY), rabbit anti-NK1R (1:2000, Advanced Targeting Systems and Millipore), rabbit anti-Pax2 (1:250, Invitrogen), goat anti-Pax $2 / 5 / 8$ (1:100, SCBT), goat anti-Phox $2 \mathrm{~b}(1: 500$, SCBT), rabbit anti-Phox $2 \mathrm{~b}$ (1: 20,000, C. Gordis, École Normale Supérieure, Paris, France), goat antiSST (1:600, SCBT), rabbit anti-SST (1:500, SCBT), guinea pig antisomatostatin 2a receptor (SST2aR) (1:8000, Gramsch Labs), sheep anti-tyrosine hydroxylase/tryptophan hydroxylase (1:1000, Millipore). Secondary antibodies were species specific and conjugated to AMCA, FITC, Alexa 488, DyLight 488, Cy3, C5, or DyLight 649 (Invitrogen or Jackson ImmunoResearch).

In situ hybridization. Slides were immersed in $4 \%$ PFA, permeabilized with proteinase $\mathrm{K}$, washed in $0.1 \mathrm{~m}$ triethanolamine- $\mathrm{HCl}$ with $0.25 \%$ acetic anhydride, blocked in hybridization buffer at $65^{\circ} \mathrm{C}$, then placed into slide mailers containing hybridization buffer with DIG-labeled antisense RNA at $1 \mu \mathrm{g} / \mathrm{ml}$ overnight at $65^{\circ} \mathrm{C}$. Slides were washed in SSC buffers at $62^{\circ} \mathrm{C}$, then washed and incubated in alkaline phosphataseconjugated anti-DIG antibody in 10\% NHS and incubated in NBT-BCIP until cellular labeling is clear. For combined immunohistochemistry and in situ hybridization (ISH), slides are stained for mRNA expression before immunohistochemical labeling.

Genotyping. Mice were genotyped by PCR using primers specific for $\beta$-gal, GFP/YFP, Neomycin phosphotransferase, Cre recombinase, Atoh1, Dbx1, and Lmx1b as described previously (Pressman et al., 2000; Pierani et al., 2001; Rose et al., 2009), or by direct visualization of fluorescent reporter, $\beta$-gal reaction product, or anatomical defect.

Tissue acquisition. Neonatal pups ( $\mathrm{P} 0-\mathrm{P} 4)$ or embryos from timed pregnant females (morning of plug $=\mathrm{E} 0.5, \mathrm{E} 10.5-\mathrm{E} 18.5)$ were anesthetized and either perfused ( $\geq$ E16.5) or immersion fixed in $4 \%$ paraformaldehyde in $0.1 \mathrm{M}$ PBS, pH 7.4. Embryos or isolated brainstems were postfixed in PFA overnight at $4^{\circ} \mathrm{C}$, cryoprotected in $25 \%$ sucrose in PBS, blocked, frozen in OCT, and stored at $-75^{\circ} \mathrm{C}$. Brainstems were sectioned in sets of six on a Hacker cryostat at $20 \mu \mathrm{m}$, and sections were thaw mounted onto Superfrost Plus slides and stored at $-20^{\circ} \mathrm{C}$ until use.

Immunohistochemical and in situ hybridization image acquisition. Fluorescent and bright-field images were acquired using a Nikon 90i microscope (Nikon Instruments), Roper $\mathrm{H} 2$ cooled CCD camera (Photometrics), and Optigrid Structured Illumination Confocal with a Prior (Rockland) motorized translation stage. Pseudocolored images were acquired in Velocity (PerkinElmer), modified in Photoshop (Adobe) or ImageJ (National Institutes of Health), and exported as 8 bit JPEG images. Images were filtered and levels were modified for clarity.

Video recording. Video images were acquired using a Nikon D90 camera with $105 \mathrm{~mm}$ Nikkor Macro lens (Nikon Instruments). Video was processed in IMovie HD (Apple Computer) and individual frames were exported to Photoshop for analysis.

BrdU and tamoxifen labeling. 5-Bromo-2-deoxyuridine (BrdU) $(50 \mathrm{mg} /$ $\mathrm{kg}$, Roche) was injected intraperitoneally into timed pregnant mice with sterile saline $(20 \mathrm{mg} / \mathrm{ml})$ as previously described (Pagliardini et al., 2003). Tamoxifen (1-2 mg, $10 \mathrm{mg} / \mathrm{ml}$ in vegetable oil) was given by oral gavage in timed pregnant mice as previously described (Hirata et al., 2009).

Cell counting. Cells or nuclei larger than $5 \mu \mathrm{m}$ in diameter were counted from the caudal end of the VII motor nucleus (VIIn) to the caudal pole of the lateral reticular nucleus (LRN) by visual inspection of confocal images acquired at $10-20 \times$ within either a $500 \mu \mathrm{m}$ circle encompassing the majority of the VLM, or a $350 \mu \mathrm{m}$ circle ventral to the nucleus ambiguus. Levels of digital images were enhanced to maximize low-level expression. The first section within a set containing the VIIn was set as $0 \mu \mathrm{m}$. Colocalization of $\beta$-Gal and mRNA required at least half of the nucleus to be surrounded.

En bloc recordings (quantal slowing). P0 to P2 CF1 mice were anesthetized by cooling and ether (Merck) and immersed in cooled $\left(4^{\circ} \mathrm{C}\right)$ artificial CSF (aCSF) containing the following (in $\mathrm{mm}$ ): $125 \mathrm{NaCl}, 5 \mathrm{KCl}$, $24-28 \mathrm{NaHCO}_{3}, 1.25 \mathrm{KH}_{2} \mathrm{PO}_{4}, 30$ D-glucose, $0.8 \mathrm{CaCl}_{2}$, and $1.25 \mathrm{MgSO}_{4}$ (Sigma). The medium was equilibrated with carbogen: $95 \% \mathrm{O}_{2} / 5 \% \mathrm{CO}_{2}$, $\mathrm{pH}$ 7.40. The CNS was removed, decerebrated through a pontomesencephalic transection, and transferred to a $2 \mathrm{ml}$ recording chamber. Newborn animals in each experimental group came from at least 3-4 litters. No pontobulbar transection was performed.

Spontaneous activity from C3-C5 ventral roots was recorded with glass suction electrodes $\left(24-25^{\circ} \mathrm{C}\right)$. Electrical signals were amplified by a low-noise differential amplifier (Grass Instruments), processed with a full-wave rectifier and leaky integrator (time constant $=100 \mathrm{~ms}$ ), displayed on a monitor, and recorded via Digidata $1320 \mathrm{AD}$ acquisition system (Molecular Devices). Analyses were performed in Axoscope software (Molecular Devices).

Somatostatin was added to the aCSF for a final concentration between $10^{-10}$ and $10^{-14} \mathrm{M}$. In six preparations, SST doses were $10^{-12} \mathrm{M}$ with slight visualization of quantal slowing. Quantal slowing was observed in four of six preparations using SST doses between $10^{-13} \mathrm{M}$ and $10^{-14} \mathrm{M}$.

En bloc recordings (Dbx1 mutant mice). E18.5 Dbx1 ${ }^{\mathrm{LacZ} /+}$ and Dbx1 $1^{\text {LacZ/LacZ }}$ were removed by cesarean section from anesthetized timed-pregnant animals. Brainstem-spinal cord preparations extending from the pontomedullary junction to sacral region of the spinal cord were isolated by craniotomy and laminectomy from pups, deeply anesthetized by cooling, and immersed in cold $\left(4^{\circ} \mathrm{C}\right) \mathrm{aCSF}, \mathrm{pH} 7.4$, containing the following (in mM): $124.0 \mathrm{NaCl}, 5.0 \mathrm{KCl}, 2.4 \mathrm{CaCl}_{2}, 1.3 \mathrm{MgSO}_{4}, 26.0 \mathrm{NaHCO}_{3}, 1.2$ $\mathrm{KH}_{2} \mathrm{PO}_{4}$, and 30 glucose equilibrated with $95 \% \mathrm{O}_{2}$ and $5 \% \mathrm{CO}_{2}$ to $\mathrm{pH}$ 7.4. These preparations were incubated at $25-26^{\circ} \mathrm{C}$ for up to $2-3 \mathrm{~h}$ under constant aeration and transferred to a $6 \mathrm{ml}$ recording chamber.

Simultaneous spontaneous activity from cervical C2-C5 ventral roots was recorded with glass suction electrodes. Electrical signals were amplified by a low-noise differential amplifier (Grass Instruments). The signals were digitally acquired using an analog-to-digital converter (ADInstruments). LabChart 7 Pro (v7.1.2, ADInstruments) software was used for signal processing.

Serotonin $(5 \mu \mathrm{M})$ and SST $\left(10^{-13} \mathrm{M}\right)$ were added to aCSF and bath applied to the entire preparation. Low $\mathrm{pH}$ (7.0) aCSF contained the following (in $\mathrm{mm}$ ): $140.0 \mathrm{NaCl}, 5.0 \mathrm{KCl}, 2.4 \mathrm{CaCl}_{2}, 1.3 \mathrm{MgSO}_{4}, 10.0 \mathrm{NaHCO}_{3}, 1.2$ $\mathrm{KH}_{2} \mathrm{PO}_{4}$, and 30 glucose equilibrated with $95 \% \mathrm{O}_{2}$ and $5 \% \mathrm{CO}_{2}$. 
Slice recordings. Transverse slices (550 $\mu \mathrm{m}$ thick) from neonatal (P0P6) Dbx $1^{\text {LacZ }}$ and Dbx1 ${ }^{\text {CreERT2 }}$; $26 \mathrm{R}^{\text {EYFP }}$ mice were dissected and prepared for recordings as described previously (Hayes and Del Negro, 2007). On-cell and whole-cell patch recordings were obtained using infrared-enhanced differential interference contrast (IR-DIC) videomicroscopy after fluorescent identification of $\mathrm{Dbxl}^{+}$neurons in both mouse models. Slices from $\mathrm{Dbx} 1^{\mathrm{LacZ} /+}$ mice were incubated with fluorescein-di- $\beta$-D-galactopyranoside (FDG). Cleavage of this nonfluorescent sugar moiety in $\beta$-gal-expressing cells releases fluorescein. Slices from Dbx1 ${ }^{\text {CreERT2 }}$;R26R ${ }^{\text {EYFP }}$ showed robust reporter expression in the preBötC when tamoxifen was administered on E10.5. aCSF contained the following (in mM): $124 \mathrm{NaCl}, 9 \mathrm{KCl}, 0.5 \mathrm{NaH}_{2} \mathrm{PO}_{4}, 25 \mathrm{NaHCO}_{3}, 30$ D-glucose, $1.5 \mathrm{CaCl}_{2} \cdot 2 \mathrm{H}_{2} \mathrm{O}$, and $1 \mathrm{MgSO}_{4}$. Slices were placed into a $0.5 \mathrm{ml}$ chamber within an upright fixed-stage microscope (Zeiss Microimaging), and aCSF was perfused at $\sim 5 \mathrm{ml} / \mathrm{min}$ at $27-28^{\circ} \mathrm{C}$.

Patch recordings used a HEKA EPC-10 patch-clamp amplifier and Patchmaster software. Respiratory-related motor output was monitored from XII nerves with extracellular suction electrodes and a high-gain differential amplifier with bandpass filtering $(0.3-1 \mathrm{kHz})$ (Dagan Instruments), full-wave rectified, and smoothed for display. Data were acquired using Chart 5 software and a Powerlab 4/30 (ADInstruments). An $8 \mathrm{mV}$ liquid junction potential was corrected online in both current and voltage clamp.

We used a patch solution containing the following (in $\mathrm{mm}$ ): 140 K-gluconate, $5 \mathrm{NaCl}$, 0.1 EGTA, $10 \mathrm{HEPES}, 2 \mathrm{Mg}$-ATP, and $0.3 \mathrm{Na}_{3}$-GTP $(\mathrm{pH}=7.2$ using $\mathrm{KOH})$. We added $2-4 \mu \mathrm{l} / \mathrm{ml}$ of Alexa Fluor 568 hydrazide $\left(\mathrm{Na}^{+}\right.$salt, Invitrogen) to the patch solution for fluorescent visualization of morphology.

Neurons were visually identified using both IR-DIC videomicroscopy and epifluorescence illumination (X-Cite 120, EXFO) and a GFP filter to identify fluorescein- and EYFP-labeled cells. Images were acquired of recorded Dbx1 neurons. In most cases, a MaiTai Ti:sapphire ultra-fast pulsed laser (Spectra Physics) tuned to $800 \mathrm{~nm}$ was used for two-photon FDG imaging and transmitted light images (i.e., Fig. 5), and a $543 \mathrm{~nm}$ $\mathrm{HeNe}$ laser was used for confocal imaging of Alexa 568. Images were contrast enhanced and pseudocolored, and a $0.5-\mu \mathrm{m}$-radius Gaussian blur was applied using ImageJ software.

Statistics. All the Dbx1-derived neuron recordings were used to compute mean and SEM for burst amplitude, burst area, half-width (i.e., duration at $50 \%$ burst amplitude), and the preinspiratory burst latency. The latency was determined from the point of first depolarization and/or summating EPSPs above baseline to the maximum slope onset of the drive output from XII, which marks the inspiratory burst per se. Data from Dbx1 ${ }^{\text {LacZ/+ }}$ and Dbx $1^{\text {CreERT2 }} ; \mathrm{R}_{26 \mathrm{R}^{\text {EYFP }}}$ animals were similar, so the samples ( $n=8$ and $n=5$, respectively) were pooled. Cell counts were compared using a two-tailed, unequal-samples Student's $t$ test in Numbers (Apple Computer).

\section{Results}

To identify transcription factors that might define subpopulations of preBötC neurons, we analyzed the expression, by ISH, of $>75 \%(\sim 1000)$ of all transcription factors (TFs) in E13.5 and P0 mouse brain (Gray et al., 2004). While no single TF was specific to the preBötC, we identified three candidate TFs that were expressed by subsets of VLM neurons: Lhx9, Pax2, and Phox $2 \mathrm{~b}$. Using immunohistochemistry (IHC), we analyzed Lhx9, Pax2, and Phox $2 \mathrm{~b}$ coexpression with NK1R or SST throughout the VLM. In the neonate mouse, NK1R expression is continuous from the caudal pole of the LRN extending beyond the VIIn, including the neurons of the pFRG and the RTN (Gray et al., 1999; Stornetta et al., 2006; Rose et al., 2009; Thoby-Brisson et al., 2009). Thus NK1R expression alone does not identify the boundaries of the preBötC in neonatal mice. SST protein or preprosomatostatin mRNA (data not shown) were limited to the preBötC, similar to rat (Stornetta et al., 2003). SST largely colocalizes with NK1R $(87.3 \%, 144 / 165, n=4)$, which suggests that it can serve as an anatomical marker of the "core" of the preBötC in mice (Fig.
$1 B, C-E$ ) (Stornetta et al., 2003; Tan et al., 2008). We found that a subset of Pax2-expressing neurons coexpress SST within the preBötC (Fig. 1A). Lhx9 and Phox 2 b were both expressed throughout the VLM (Gray, 2008); however, they were coexpressed with NK1R only outside the preBötC (supplemental Fig. 1, available at www.jneurosci.org as supplemental material), and neither was coexpressed with SST. Pax 2 did not overlap with Phox $2 \mathrm{~b}$ or Lhx9 anywhere in the medulla.

\section{PreBötC neurons are derived from Dbx1-expressing progenitors} Adult rat preBötC SST ${ }^{+}$neurons are glutamatergic and commissural (Guyenet and Wang, 2001; Stornetta et al., 2003). In the spinal cord, only Dbx1-derived neurons express Pax2, are glutamatergic, and project across the midline (Lanuza et al., 2004). This suggested that preBötC SST neurons might be derived from Dbx1-expressing progenitors (Lanuza et al., 2004). Dbx1 is only expressed in progenitor cells, whereas NK1R and SST expression in preBötC does not commence until E14.5-E15.5, so we could not directly analyze Dbx1 coexpression with these markers ( $\mathrm{Pa}$ gliardini et al., 2003; Thoby-Brisson et al., 2005).

In heterozygous Dbx1- $\beta$-gal knock-in mice ( $\left.\mathrm{Dbx} 1^{\mathrm{LacZ} /+}\right)$ (Pierani et al., 2001), in which the persistence of the $\beta$-Gal protein can be used to fate map neurons, we found that the preBötC core population of $\mathrm{NK}_{1}{ }^{+} / \mathrm{SST}^{+}$neurons are derived from a subset of Dbx1-expressing progenitors (Fig. $1 B-H$ ). $\beta$-Gal was absent, however, from VLM NK1R ${ }^{+}$motoneurons, below the central ventrolateral medulla, and putative RTN/pFRG neurons (supplemental Fig. 2, available at www.jneurosci.org as supplemental material) (Nattie and Li, 2002, 2006; Wang et al., 2003; Takakura et al., 2008; Rose et al., 2009).

As NK1R expression is present on neurons from multiple developmental origins, we determined the extent to which VLM SST neurons are Dbx1 derived. We counted $\beta$-Gal, SST, and colocalized immunoreactive neurons in a $500-\mu \mathrm{m}$-diameter circle encompassing the VLM from the caudal pole of the VIIn to the caudal pole of the LRN. While SST immunoreactivity likely underestimates the number of neurons expressing SST mRNA (Stornetta et al., 2003), it can serve as simple and reproducible marker of neurons. Interestingly, the number of $\beta-\mathrm{Gal}^{+}$cells was approximately constant throughout the VLM. SST expression, in contrast, was limited rostrocaudally to an $\sim 250$ - $\mu \mathrm{m}$-long region (Fig. $1 I, J$ ). Of VLM SST neurons, $92.7 \%$ coexpressed $\beta$-Gal $(433 / 467, n=16)$. Further, $\mathrm{SST}^{+}$neurons are concentrated in the center of the $\mathrm{Dbxl}^{+}$population dorsoventrally, as $82.4 \%$ $(385 / 467, n=16)$ of them are located within a $350-\mu \mathrm{m}$-diameter circle centered on SST expression. This $250 \mu \mathrm{m}$ by $350 \mu \mathrm{m}$ column represents the anatomical extent of the preBötC core in the perinatal mouse (Ruangkittisakul et al., 2006).

Differential peptide receptor expression has been proposed to distinguish between subsets of respiratory neurons (Gray et al., 1999; Doi and Ramirez, 2008, 2010; Ruangkittisakul et al., 2008; Ballanyi et al., 2009). In rats, injection of somatostatin at high doses into the preBötC inhibits respiration (Burke et al., 2010). Because of the inhibitory effects of SST in vivo and in vitro, SST coexpression in preBötC neurons, and previously published expression patterns, we also analyzed SST2aR expression within the preBötC and ventral medulla (Schindler et al., 1998; Schulz et al., 2000; Llona and Eugenín, 2005; Burke et al., 2010). SST2aR is expressed throughout the VLM (Schindler et al., 1998). Within the preBötC, SST2aR is coexpressed with SST peptide and NK1R in Dbx1-derived neurons (Fig. 1B). Unlike NK1R, however, SST2aR is also expressed on catecholaminergic neurons (data not shown) but is not expressed on RTN/pFRG neurons (supple- 
mental Fig. 1C,D, available at www. jneurosci.org as supplemental material). These data suggest that SST2aR, similar to $\mu$-opioid receptor, may serve to functionally and anatomically differentiate preBötC from RTN/pFRG neurons (Takeda et al., 2001; Mellen et al., 2003).

Within the preBötC core of Dbx1 ${ }^{\mathrm{LacZ} /+}$ mice, $\beta$-Gal, SST peptide, NK1R, and SST2aR partially colocalize (Fig. $1 B-H$ ); $86.6 \%(129 / 149, n=4)$ of $\beta-\mathrm{Gal}^{+} \mathrm{SST}^{+}$ neurons coexpress NK1R (Fig. $1 C-E$ ). Unlike $\mathrm{SST}^{+}$neurons, Dbx1-derived NK1R or SST2aR-expressing neurons are not limited to the preBötC core. Outside this region, there is extensive heterogeneity of receptor expression in the Dbxl-derived population (supplemental Fig. 2, available at www.jneurosci.org as supplemental material). For example, within the BötC, there is little $\beta-\mathrm{Gal} / \mathrm{SST} 2 \mathrm{aR}$ coexpression, but clear $\beta$-Gal/NK1R coexpression extending up to the VIIn boundary (Fig. $1 \mathrm{H}$; supplemental Fig. $2 B, C$, available at www. jneurosci.org as supplemental material). Even within the preBötC core, only $17.0 \%$ $(149 / 875, n=4)$ of $\beta-\mathrm{Gal}^{+}$cells coexpressed SST, and $21.6 \%(474 / 2191, n=8)$ coexpressed NK1R. In the VLM as a whole, SST represented only $4.9 \%$ of $\beta$-Gal-labeled cells $(433 / 8748, n=16)$. Moreover, at least 44.2\% (3868/8748, $n=$ 16) of VLM Dbx1-derived cells lie outside the preBötC core. This demonstrates that Dbx1-derived neurons comprise a larger percentage of putative respiratory neurons in the VLM, which includes, but is not limited to, the preBötC.

\section{VLM Dbx1 neurons are glutamatergic}

In other regions of the CNS, Dbx1 proliferative zones generate glutamatergic, GABAergic, and cholinergic neurons, as well as a subset of glia (Lanuza et al., 2004; Bielle et al., 2005; Fogarty et al., 2005; Zagoraiou et al., 2009). We used ISH and IHC to determine the cellular and transmitter identity of Dbx $1 \beta-\mathrm{Gal}^{+}$cells in the VLM. At least 44.7\% (1630/3645, $n=9)$ of VLM Dbx1 $\beta$-Gal ${ }^{+}$cells coexpressed mRNA for VGlut2, a marker of glutamatergic neural identity (Fig. $2 A$ ), and $20.7 \%$ $(533 / 2577, n=5)$ of $\beta$-Gal ${ }^{+}$cells coexpressed $\mathrm{S} 100 \beta$ protein, a marker of glial lineage (Fig. $2 E$ ). In contrast, very few VLM Dbx $1 \beta$-Gal cells were inhibitory, with only $2.8 \%(16 / 562, n=3)$ coexpressing glutamic acid decarboxylase 1 mRNA (GAD1), a marker of GABAergic neurons (Fig. 2 B), 4.8\% (54/1118, $n=7)$ of $\beta$-Gal cells coexpressing vesicular glycine transporter (Slc6a5) mRNA, a marker of glycinergic neurons (Fig. 3C), and 2.4\% (53/2146, $n=5$ ) of $\beta$-Gal cells coexpressing choline acetyltransferase
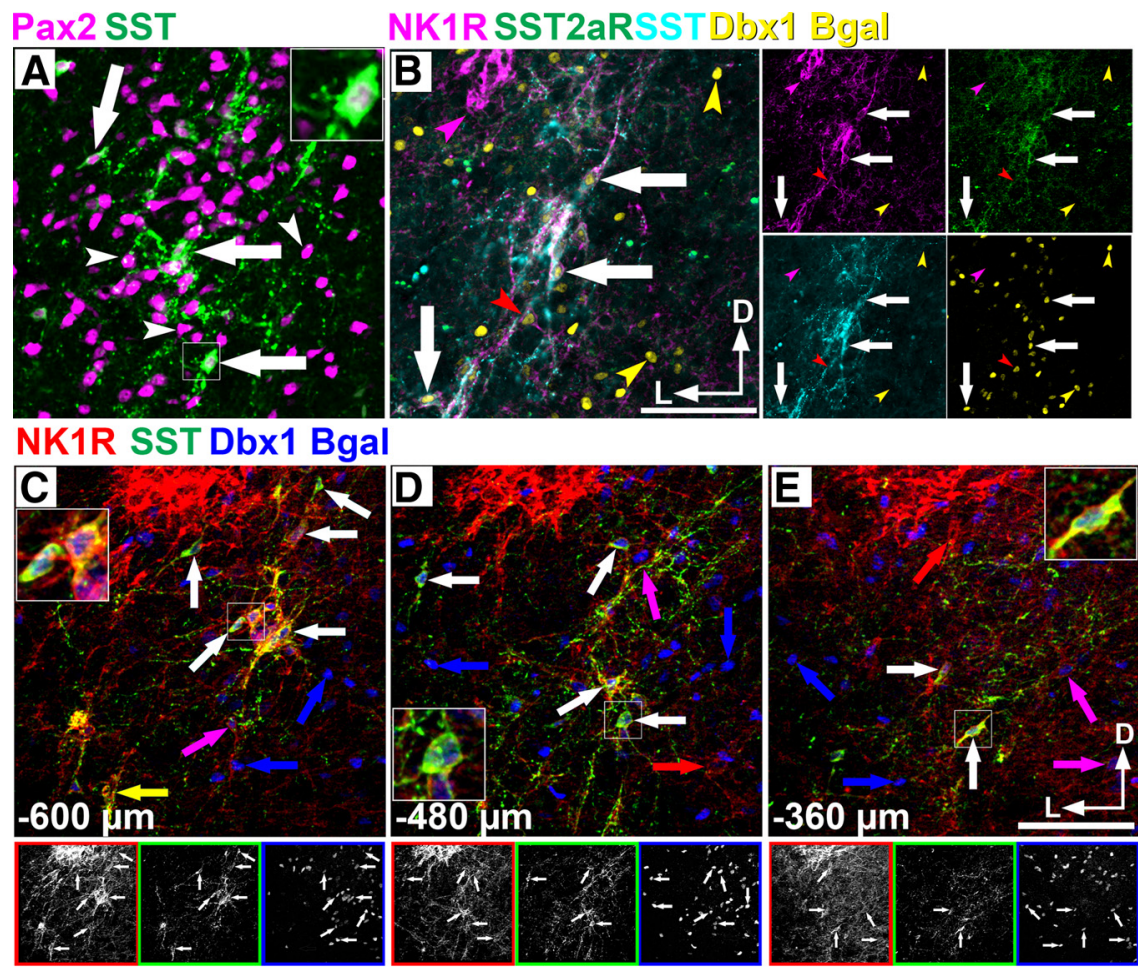

NK1R SST2aRDbx1 Bgal
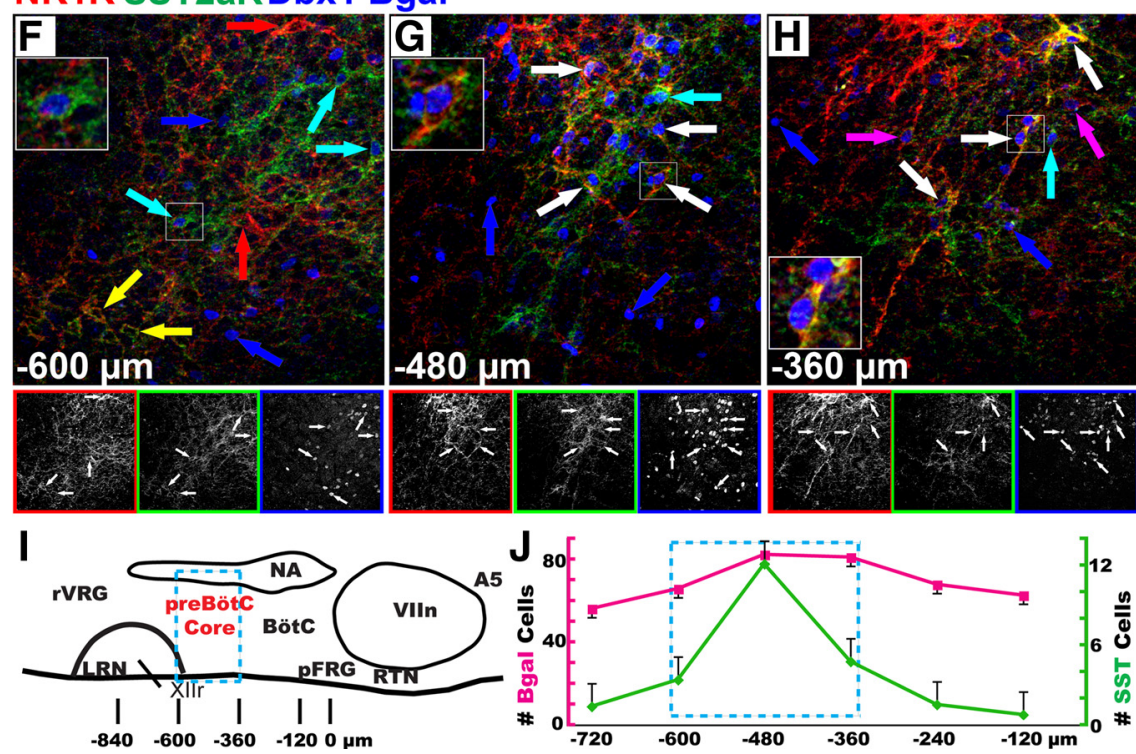

Figure 1. Mouse preBötzinger Complex neurons are Dbx1 derived. $\boldsymbol{A}$, Confocal pseudocolor images of SST (green) and Pax2 (magenta) coexpression within the mouse preBötC. Arrows indicate coexpression. White arrowheads indicate absence of twogene coexpression. $\boldsymbol{B}$, Coexpression of NK1R (magenta), SST2aR (green), SST (cyan), and $\beta$-Gal (yellow) in Dbx $1^{\text {LacZ/+ }}$ preBötC. Lateral images correspond to single-channel images. Colored arrowheads indicate single gene expression. $\mathbf{C}-\boldsymbol{H}$, Partially convergent peptidergic signaling in the mouse preBötC. Confocal pseudocolor images showing the heterogeneity of NK1R (red, $\mathbf{C}-\boldsymbol{H}$ ), SST (green, $\boldsymbol{C}-\boldsymbol{E}$ ), SST2aR (green, $\boldsymbol{F}-\boldsymbol{H}$ ), and Dbx1 $\beta$-Gal (blue, $\boldsymbol{C}-\boldsymbol{H})$ coexpression within the perinatal mouse preBötC between -600 and $-360 \mu \mathrm{m}$ caudal to the VIIn in the neonatal mouse. Lower images correspond to single-channel images $(\boldsymbol{C}-\boldsymbol{H})$. Large insets correspond to area in small squares. Colored arrows indicate partial coexpression. White arrows indicate complete coexpression. $\boldsymbol{A}-\boldsymbol{H}$ are from PO mice. I, Diagram representing sagittal view through the respiratory column (rostral right) of the neonatal mouse brainstem and relative location of respiratory populations and locations of staining in $\mathbf{C} \boldsymbol{H}$. J, Graph showing number of $\beta$-Gal (magenta, left) or SST (green, right) cells per hemisection within a $500 \mu \mathrm{m}$ circle encompassing the VLM by distance caudal to the VIIn. Cyan box $(I, J)$ corresponds to the "preBötC core region." Scale bar, $200 \mu \mathrm{m}$. D, Dorsal; L, lateral; A5, A5 region; XIIr, level of exit of XII motor root from the ventral hindbrain.

(ChAT) protein, a marker of cholinergic neurons (Fig. $2 D$ ). In general, the inhibitory Dbx1 $\beta$-Gal neurons were dorsomedial to the VLM (Fig. $2 B, C$ ). Adjacent to the XII motor nucleus, there was extensive overlap of $\beta$-Gal with GAD1 or VGlut2 

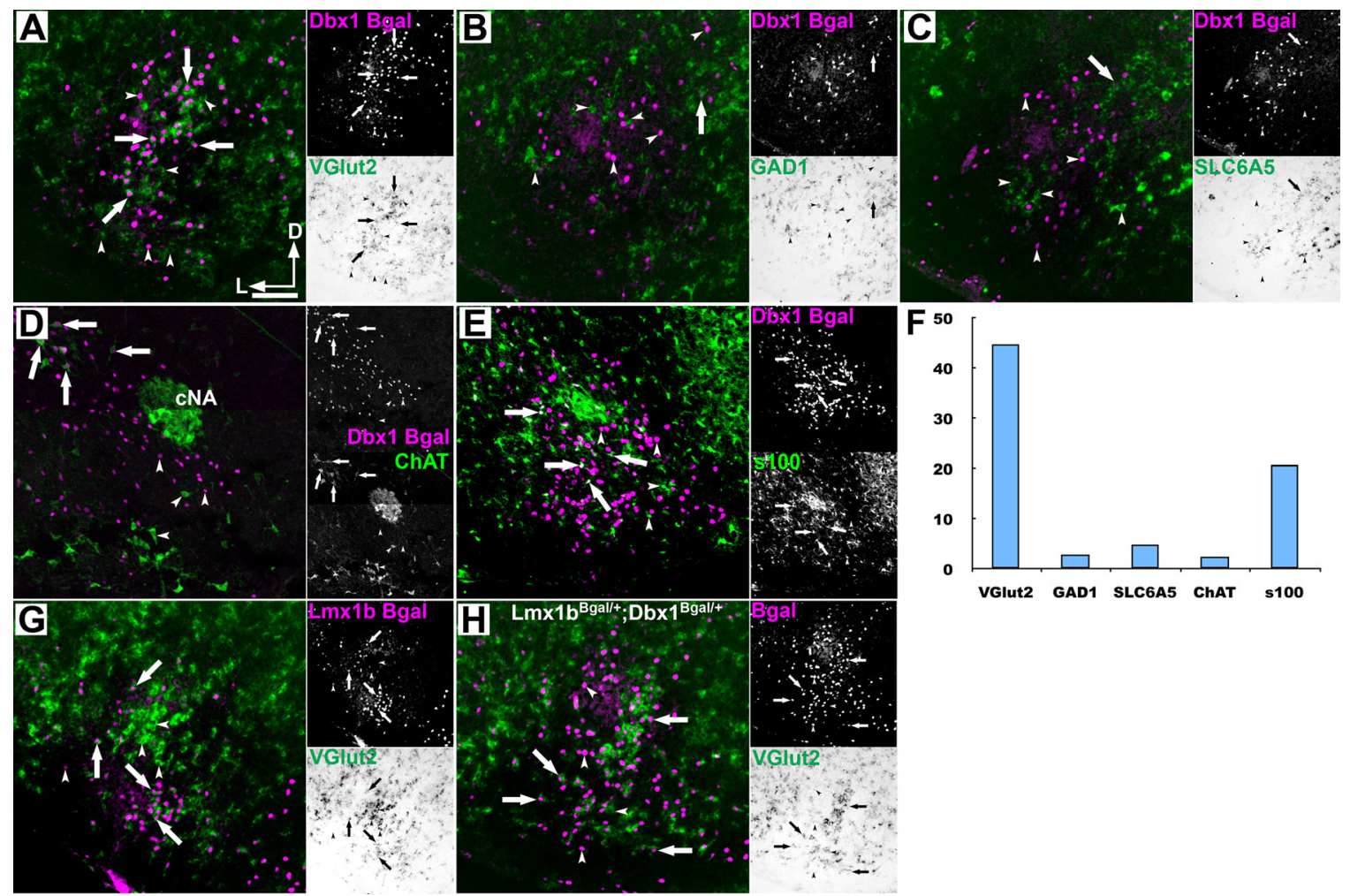

Figure 2. Ventral medullary respiratory glutamatergic neurons are Dbx1 derived. $\boldsymbol{A}-\boldsymbol{E}, \mathbf{G}, \boldsymbol{H}$, Pseudocolor mosaic images of $\beta$-Gal (magenta) $\mathrm{IHC}_{\mathrm{from}} \mathrm{Dbx} 1^{\mathrm{Lac} Z /+}(\boldsymbol{A}-\boldsymbol{E}), \mathrm{Lmx} 1 \mathrm{~b}^{\mathrm{Lac} Z /+}(\boldsymbol{G})$, or

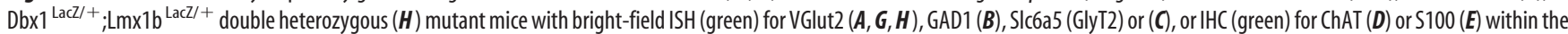
preBötC $(\boldsymbol{A}-\boldsymbol{C}, \boldsymbol{E})$ or adjacent to the cNA (D). Lateral images correspond to single-channel images. $\boldsymbol{F}$, Percentage of $\beta$-Gal cells in the VLM that coexpress specific genes. Arrows point out coexpression. Arrowheads indicate absence of coexpression. Scale bar, $200 \mu \mathrm{m}$. D, Dorsal; L, lateral; cNA, compact formation of nucleus ambiguus.

mRNA, consistent with spinal cord expression (Lanuza et al., 2004). The remaining $\sim 25 \%$ of $\beta$-Gal cells likely include S100 $\beta$-negative oligodendrocytes (Fogarty et al., 2005) and neurons with levels of transmitter marker mRNA below threshold for detection.

As combined ISH and IHC protocols limit sensitivity, we also analyzed the coexpression of Dbx1 $\beta$-gal in double transgenic mice in which GFP was driven with either a Slc6a5 or GAD1 promoter (Gong et al., 2003; Chattopadhyaya et al., 2004; Winter et al., 2007; Morgado-Valle et al., 2010; Winter et al., 2010). The expression patterns of GFP in both lines were consistent with both ISH and TF expression in the developing and perinatal mouse brainstem. Consistent with our ISH analysis, we found little overlap of $\beta$-gal with GFP in Dbx1 ${ }^{\mathrm{LacZ} /+}$;Slc6a5 ${ }^{\mathrm{GFP}}$ mice (supplemental Fig. 3B, available at www.jneurosci.org as supplemental material), and overlap of $\beta$-gal with Dbx1 ${ }^{\text {LacZ/+ }}$;Gad $1^{\text {GFP }}$ near the midline (supplemental Fig. $3 A$, available at www. jneurosci.org as supplemental material). In the VLM, however, we found no overlap of $\beta$-gal, NK1R, or SST2aR with GFP in either transgenic line (Fig. $2 B, C$ ).

Glutamatergic neurotransmission is essential for respiratory rhythm generation both in vivo and in vitro (Wallén-Mackenzie et al., 2006). Many current models of the respiratory network hypothesize the preBötC contains two distinct subpopulations of glutamatergic neurons. A subset of specialized neurons that generate the rhythm, and a second population of "follower" neurons thought to play a role in shaping the pattern of motor output (Feldman and Smith, 1989; Feldman et al., 1990; Butera et al., 1999; Koshiya and Smith, 1999; Del Negro et al., 2001; Ramirez and Garcia, 2007; Smith et al., 2007). Dbx1-derived neurons, however, only account for a subset of VLM glutamatergic neu- rons (Fig. 2A). This suggests that non-Dbx1-derived neurons might play an important role in breathing. Previous work has shown that nonrespiratory VLM catecholaminergic neurons release glutamate, express VGlut2, and are derived from Lmx1bexpressing progenitors and would account for some of the remaining glutamatergic neurons (Qian et al., 2002; Stornetta et al., 2002; Gray, 2008). Similar to Dbx1 neurons, we analyzed the coexpression of VGlut2 in the VLM of Lmx1b ${ }^{\text {LacZ/+ }}$ mutant mice (Pressman et al., 2000). The bulk of Lmxlb-derived catecholaminergic, glutamatergic neurons were concentrated ventral to the Dbxl-derived population, but like Dbxl were found throughout the VLM (Fig. $2 G$ ). To better estimate the extent to which VLM glutamatergic neurons were derived from Dbx1 and Lmx1b precursors, we generated Dbx ${ }^{\mathrm{LacZ} /+} ; \mathrm{Lmx} 1 \mathrm{~b}^{\mathrm{LacZ} /+}$ double heterozygous mutant mice. We found that these two developmentally defined populations account for nearly all VLM glutamatergic neurons within the preBötC and adjacent catecholaminergic population (Fig. $2 H)(n=2)$. This suggests that the majority of VLM glutamatergic neurons concerned with breathing are derived from the single Dbxl progenitor domain.

\section{Developmental origin of VLM Dbx1 neurons}

As only a spatially clustered subset of Dbx1-derived neurons coexpress SST, we wondered whether they represented a developmentally unique subpopulation. To test whether preBötC SST neurons were born at a different time than surrounding SST neurons, we injected pregnant $\mathrm{Dbx} 1^{\mathrm{LacZ} /+}$ mice with a single dose of BrdU on E9.5, E10.5, or E11.5, to label dividing cells, and counted the number of $\beta$-Gal-, BrdU-, and SST-labeled cells in P0 animals (Fig. 3A-C,E). With injection at E9.5, 21\% (474/2252, $n=4$ ) of VLM $\beta$-Gal neurons coexpress BrdU, while $21.4 \%$ 
$(40 / 187, n=4)$ of VLM SST neurons coexpressed BrdU. With E10.5 injection, $\beta-\mathrm{Gal} / \mathrm{BrdU}$ coexpression was 33\% (1213/ $3678, n=5)$ and SST/BrdU coexpression was $28.7 \%(47 / 164, n=6)$. With E11.5 injection, $\beta-\mathrm{Gal} / \mathrm{BrdU}$ coexpression was $17.4 \%(500 / 2878, n=4)$ and SST/BrdU coexpression was $9 \%(33 / 367, n=6)$. This latter difference is likely due to the onset of gliogenesis by E11.5 (Fogarty et al., 2005). These data suggest that preBötC SST-expressing neurons are born in the same proportion as non-SST-expressing neurons.

As the Dbx1 ${ }^{\text {LacZ }}$ mouse is a heterozygous mutation, it is possible that the timing of neural specification might be affected. To test this, we examined the coexpression of SST and NK1R in a Dbx1 ${ }^{\text {CreERT2 }}$ knock-in mouse with no loss of Dbxl gene function (Hirata et al., 2009). Crossing this mouse with a Rosa26-EYFP (R26R ${ }^{\text {YFP }}$ ) (Srinivas et al., 2001) line, we find that application of tamoxifen at E9.5 labels the majority of preBötC NK1R ${ }^{+} / \mathrm{SST}^{+}$neurons, which is consistent with our previous results (Fig. 3D) $(n=5)$. Together these data suggest that SST expression with the subset of Dbxl neurons is not a consequence of time of birth.

While the basic dorsoventral development of the hindbrain resembles that in spinal cord (Gray, 2008), we sought to determine whether hindbrain Dbx1 neurons were derived from the V0 progenitor domain (Pierani et al., 2001; Lanuza et al., 2004). As expected, Dbx1-derived cells of the caudal hindbrain were limited to the V0 progenitor domain, as $\beta$-gal cells are bounded by bHLHb5 expression in the DB4 and V1 progenitor domains (Fig. $3 F, G$ ) (Liu et al., 2007; Gray, 2008). Consistent with previous reports, NK1R expression in the preBötC was first evident in a small population of cells near the nucleus ambiguus (NA) at E14.5 (Fig. 3H), and SST protein expression is first evident at E15.5 (data not shown) (Pagliardini et al., 2003; Thoby-Brisson et al., 2005). In the spinal cord, Evx1 defines a specific subpopulation of V0 interneurons. After E13.5 in the hindbrain, however, Evx1 mRNA and protein are no longer specific to the V0 lineage (data not shown), preventing our ability to determine V0 subtype-specific origin of preBötC neurons (Lanuza et al., 2004).

Somatostatin induces "quantal slowing" in en bloc preparations Although the relationship between Dbx1-derived neurons of the VLM and respiratory behavior is unclear, the coexpression of SST and SST2aR within preBötC Dbx1-derived neurons, but not more rostral RTN/pFRG populations, suggests that SST might selectively modulate preBötC neurons in a manner similar to opioids in the rat brain (Gray et al., 1999; Mellen et al., 2003). Although other subclasses of SST receptors besides SST2aR have
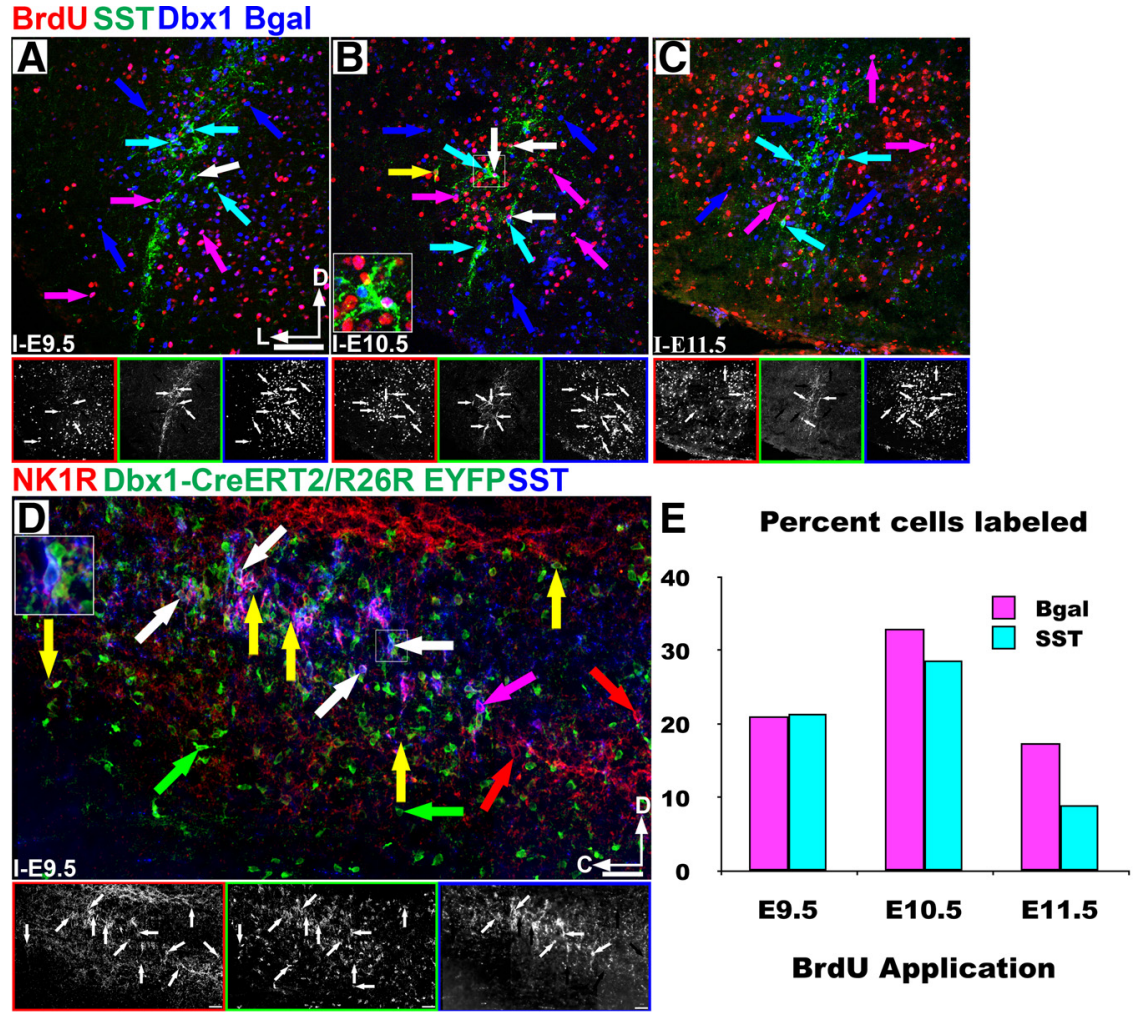

BrdU Application

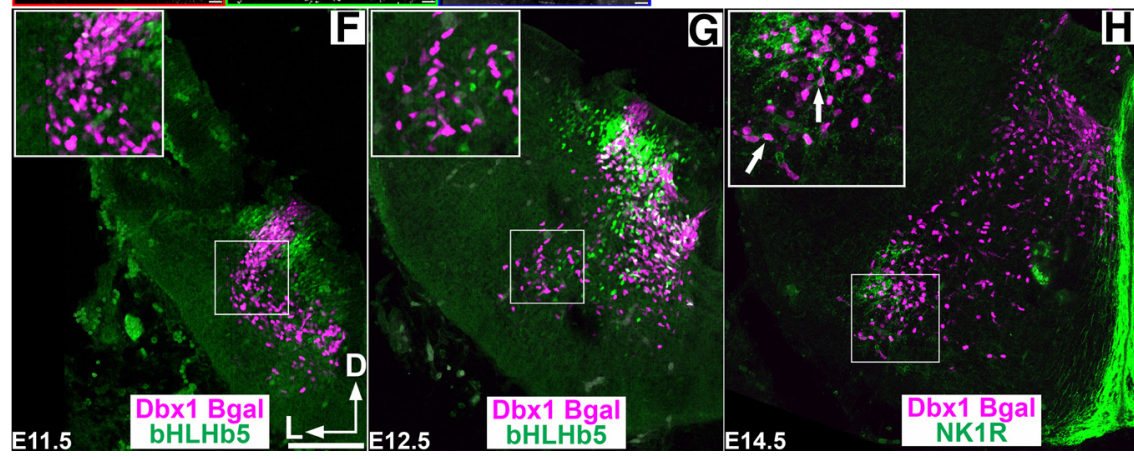

Figure 3. PreBötC core neurons are not organized by temporal birth. $A-C$, Pseudocolor mosaic images in the PO preBötC showing coexpression of BrdU, SST, and Dbx1 $\beta$-Gal (red, green, blue) after injection at E9.5-E11.5. Lower images correspond to single-channel images. D, Coexpression of NK1R (red) and SST (blue) in PO Dbx1 ${ }^{\text {CreERT2 } / R 26 R}{ }^{\text {EYFP }}$ in sagittal section through the E9.5 tamoxifen application. Note the rostrocaudal extent of coexpression. Large insets $(\boldsymbol{B}, \boldsymbol{D})$ correspond to area in mall squares. White arrows indicate triple coexpression, and colored arrows indicate single or double expression. $E$, Graph show the percentage of Dbx1 $\beta$-Gal (magenta) or Dbx1 $\beta$-Gal/SST double-labeled cells (cyan) that coexpress BrdU with injections .5-E11.5. F, G, Dbx1 $\beta$-Gal-expressing cells are derived from the V0 progenitor domain, as $\beta$-Gal (magenta) expression is bounded by DB4 and V1 populations labeled with bHLHb5 (green) at E11.5-E12.5. $\boldsymbol{H}$, NK1R coexpression (green) begins at E14.5 in mouse Dbx1 $\beta$-Gal cells. Insets show larger scale of boxed area. Scale bar, $200 \mu \mathrm{m}$. D, Dorsal; L, lateral; C, caudal.

been identified in the brainstem, we predicted that if Dbx1derived neurons are an essential part of the respiratory network, their partial inhibition by SST should produce "quantal slowing" of the in vitro respiratory period of isolated mouse en bloc preparations (Mellen et al., 2003; Janczewski and Feldman, 2006a,b).

When extremely low concentrations of SST were used (Buscail et al., 1994), the periods of fictive respiratory output clustered near integer multiples of the period before drug application. These data were similar to the effects of low concentrations of opioids in rat (Mellen et al., 2003). Figure 4 shows the effects of $10^{-13} \mathrm{M}$ SST on fictive inspiratory motor output en bloc from a P0 mouse displayed as raw traces $(A)$ and plotted on a cycle-to-cycle basis $(B)$. A histogram of normalized periods from four different en bloc preparations $\left(10^{-13}\right.$ to $10^{-14} \mathrm{M}$ SST $)$ demonstrates the 

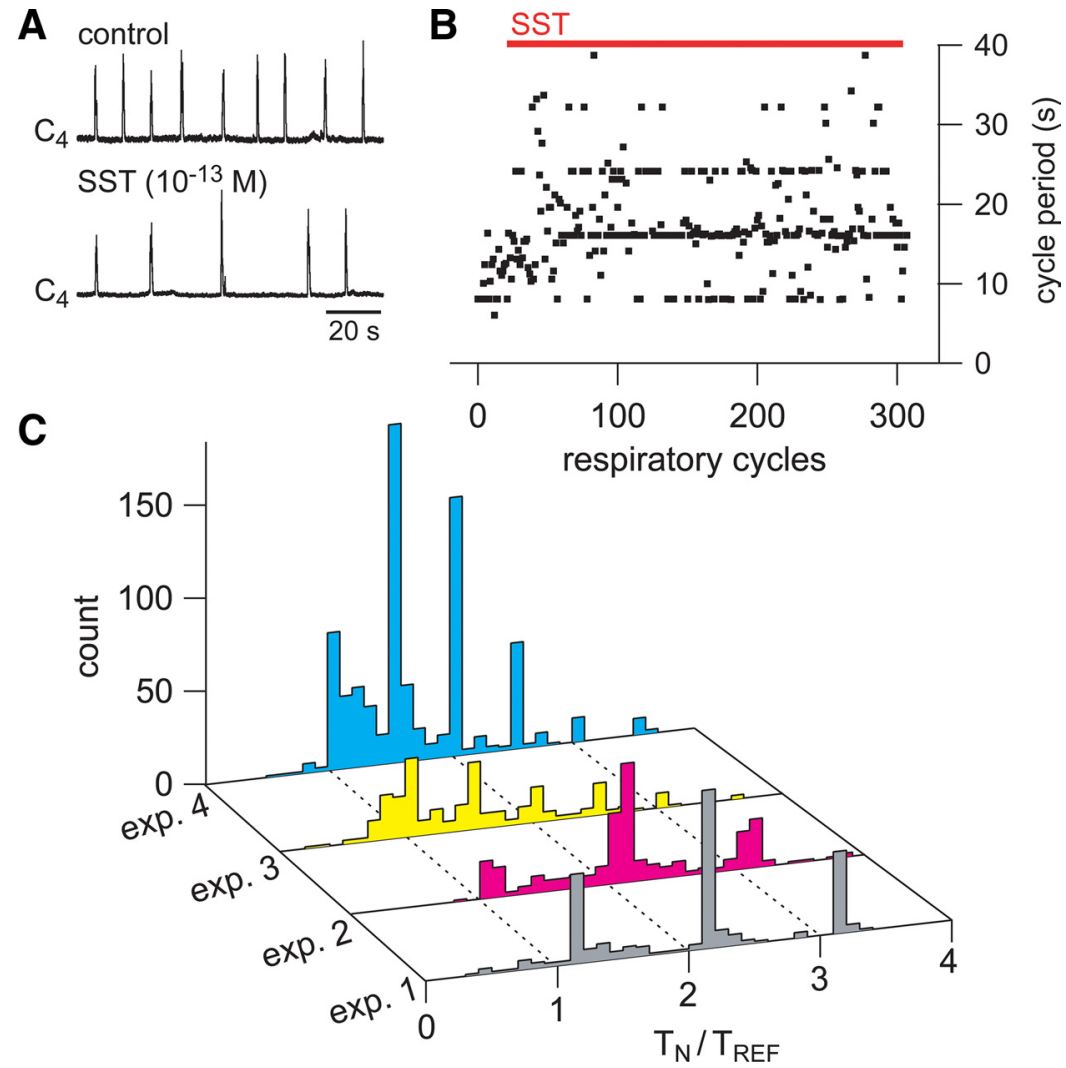

Figure 4. Low dose SST produces "quantal slowing" of fictive inspiration. $A$, Integrated fictive inspiratory output of C 4 before (control) and after bath application of $10^{-13} \mathrm{M}$ somatostatin. $\boldsymbol{B}$, Instantaneous inspiratory period before and during SST application in same preparation as $\boldsymbol{A}$. Note the clustering of periods at near-integer multiples of the control baseline period. $\boldsymbol{C}$, Period histograms of four different preparations in the presence of $10^{-13}$ M SST showing "quantal" slowing. Tn, Duration of a cycle; Tref, duration of a control cycle. ral summation of EPSCs in voltage clamp (supplemental Fig. 4D, available at www. jneurosci.org as supplemental material). The duration of the preinspiratory active phase in Dbx1 neurons measured $377 \pm$ $114 \mathrm{~ms}$ (mean \pm SEM) with a range of 129-1269 ms (193 total cycles of respiratory activity recorded in 15 cells). Inspiratory bursts were robust in Dbxl preBötC neurons, with a mean amplitude of $15.2 \pm$ $4.2 \mathrm{mV}$ (range 5-36 $\mathrm{mV}$ ) and a duration of $358 \pm 99 \mathrm{~ms}$ (range 29-1050 ms). In addition, 8 of the 15 inspiratory Dbx ${ }^{+}$ preBötC neurons exhibited characteristic inspiratory drive potentials with voltagedependent spike inactivation (i.e., depolarization block) during the bursts (Fig. $5 C)$, consistent with recruitment of the calcium-activated nonspecific cation current hypothesized to generate inspiratory bursts in preBötC neurons (Del Negro et al., 2005; Ramirez and Viemari, 2005; Rubin et al., 2009). These data show that preBötC Dbxl-derived glutamatergic neurons have a behavioral signature of firing before the onset of motor output (early I), which has been proposed as a hallmark signature of rhythmogenic function (Richter, 1982; Smith et al., 1990; Rekling et al., 1996a,b; Hayes and Del Negro, 2007). These data further suggest that Dbxl-derived neurons meet all the criteria for generating the in vitro inspiratory rhythm (Feldman and Del Negro, 2006). quantal nature of the SST modulation in four of six preparations tested (Fig. 4C). These data suggest that mouse RTN/pFRG neurons do not express functional SST receptors. They further suggest that Dbx1 neurons are functional components of the respiratory network and play a role in frequency modulation.

\section{PreBötC Dbx1-derived neurons are respiratory}

Dbxl neurons in the preBötC are strong candidates for the neurons generating the inspiratory rhythm because of their location, expression of peptide receptors, and glutamatergic phenotype. Therefore, we examined the firing patterns of Dbx1-derived neurons in rhythmic brainstem slice preparations that spontaneously generate inspiratory motor output in vitro. Fluorescent Dbx1derived neurons were selected for patch-clamp recording in $\mathrm{Dbx}^{\mathrm{LacZ} /+}(n=8)$ and Dbx1 ${ }^{\mathrm{CreERT}^{2}} ; \mathrm{R}_{26 \mathrm{R}^{\mathrm{EYFP}}}(n=8)$ mouse slices (Fig. 5A; supplemental Fig. $4 A$, available at www.jneurosci. org as supplemental material). Eighteen of the twenty neurons sampled showed rhythmic inspiratory burst activity in phase with the XII output (Fig. $5 B$; supplemental Fig. $4 B-D$, available at www.jneurosci.org as supplemental material). In addition, Dbx1 neurons were active in the preinspiratory phase $0.3-1 \mathrm{~s}$ before the XII burst (Fig. 5C). A preinspiratory active phase of duration $400 \pm 100 \mathrm{~ms}$ has been proposed as a feature of putative respiratory rhythmogenic neurons (Rekling et al., 1996a; Thoby-Brisson and Ramirez, 2001). All $18 \mathrm{Dbx}^{+}$neurons exhibited low-rate $(2-10 \mathrm{~Hz})$ spiking in current clamp (e.g., Fig. 5B; supplemental Fig. $4 B, C$, available at www.jneurosci.org as supplemental material). This preinspiratory activity was associated with the tempo-
Dbx1 is necessary for the formation of preBötC neurons

Dbx1 loss leads to the loss of a number of neural populations from the basal ganglia to the spinal cord (Pierani et al., 2001; Lanuza et al., 2004; Hirata et al., 2009). To determine the effects of the loss of Dbxl function on VLM populations, we analyzed the VLM of Dbx1 homozygous null mice (E16.5-E18.5, $n=9)$. Figure 6 shows the expression of NK1R and SST in the preBötC (panels $A$ and $B$ ) and RTN/pFRG (panel $D$ ) in an E18.5 Dbx1 ${ }^{\text {LacZ/LacZ }}$ mouse. $\beta$-gal was still present in cells of the VLM. However, NK1R- and SST-expressing neurons were completely absent from the area of the preBötC. NK1R was not coexpressed with $\beta$-gal outside of this region. NK1R expression appeared normal in the NA and RTN/pFRG (Fig. 6A,D). Additionally, SSTpositive processes and puncta were still present within the VLM, indicating that the preBötC was not the sole source of SST in this region, consistent with SST receptor expression on nonrespiratory populations (Fig. $6 \mathrm{~B}$ ). Together these data suggest that the development and expression of NK1R and other genes within the preBötC and pFRG are independently regulated.

The loss of Dbxl gene function eliminated preBötC NK1R and SST expression, but did not completely eliminate $\beta$-gal expression from the VLM. The overall number of remaining $\beta$-galexpressing cells in the VLM decreased by $39 \%$ [67.2 \pm 2.3 cells per hemisection (Dbx $1^{\mathrm{LacZ} /+}$ ), $n=14$ vs $41.5 \pm 1.9$ cells per hemisection ( $\left.\mathrm{Dbx} 1^{\mathrm{LacZ} / \mathrm{LacZ}}\right), n=5, p<0.001$, Student's $t$ test, $\mathrm{SEM}]$. In contrast, $\beta$-Gal/S100 coexpression increased by nearly $50 \%(283 / 943, n=2)$. Additionally, we found no overlap of $\beta$-gal with FoxP2 or bHLHb5, markers of V1- and DB4-derived popula- 
tions (Gray et al., 2004; Liu et al., 2007), in the VLM of either genotype, and no obvious increase in either population (data not shown). These data suggest that $\beta$-Gal expression in Dbxl ${ }^{\text {LacZ/LacZ }}$ remains an accurate representation of the Dbxl promoter activity during development.

Because the majority of VLM Dbx1derived neurons do not express SST, it was possible that the absence of peptide markers did not indicate a general loss of VLM neurons in null mice. To test this, we analyzed the expression of neurotransmitter

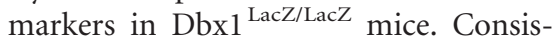
tent with the elimination of Dbx1-derived neurons, we observed a near-complete loss of VGlut 2 in the respiratory regions of the VLM as well as adjacent to the XIIn in Dbx1 $1^{\text {LacZ/LacZ }}$ mice (Fig. 6C). VGlut2expressing cells are still present in Lmxlbderived catecholaminergic neurons. In these animals, only $3.9 \%(13 / 336, n=3)$ of the remaining $\beta$-gal-expressing cells coexpressed VGlut2, while $17.9 \%$ (71/397, $n=3$, not shown) expressed GAD1. To address the possibility that Dbx1 elimination decreases $\beta$-gal expression, but does not affect other properties of neurons such as Pax2 expression, we also counted the number of VLM VGlut 2 neurons that coexpress Pax 2 but not $\beta$-gal. In Dbx $1^{\mathrm{LacZ} /+}$ animals, $82 \%(253 / 307, n=2)$ of Pax2-expressing glutamatergic neurons coexpressed $\beta$-gal. The remaining Pax $2^{+} /$VGlut $2^{+} / \beta$ $\mathrm{gal}^{-}$neurons were distributed throughout the VLM in small numbers with $1.9 \pm 0.4$ cells per hemisection. In homozygous mutants, however, the number of $\mathrm{Pax} 2^{+} / \mathrm{VGlut}^{+} / \beta$-gal ${ }^{-}$neurons per hemisection was statistically unchanged $(1.2 \pm 0.3$ cells per hemisection, $n=2, p=0.07$, Student's $t$ test), indicating that the loss of VGlut2 expression corresponds to the loss of Dbx1derived neurons. Together, these data suggest a large change in the cellular composition of the VLM in Dbxl mutant mice, which also includes an increase in the ratio of $\beta$-Gal-expressing glia and GABAergic neurons. These data further suggest that Dbxl gene function is necessary for the specification of the glutamatergic neurons of the respiratory column, including the preBötC.

\section{Dbxl is necessary for inspiration in vivo and in vitro}

E18.5 wild-type (13/13) and Dbx1 ${ }^{\text {LacZ/+ }}(14 / 16)$ mice show one or two distinct rhythmic respiratory-like behaviors within $20 \mathrm{~min}$ of cesarean delivery. The first is a classic rhythmic inspiratory activity involving activation of jaw and diaphragm muscles, expansion of the rib cage, and compression of the abdominal region, leading to inspiratory airflow (Fig. 7A; supplemental Video 1 , available at www.jneurosci.org as supplemental material). The second is a transient rhythmic activity of abdominal muscles without jaw movements, and with a slight compression of ribcage corresponding to active expiration (Fig. 7B; supplemental Video 2, available at www.jneurosci.org as supplemental material). Some animals show only inspiratory activity, while others show both, but there was no difference in either behavior between wild-type or Dbx1 ${ }^{\mathrm{LacZ} /+}$ mice. All animals exhibiting respiratory behaviors had inflated lungs upon dissection (Fig. 7C).

Dbx1 $1^{\text {LacZ/LacZ }}$ mice, in sharp contrast, do not make any inspiratory, expiratory, or jaw movements, even with extensive tac-
B

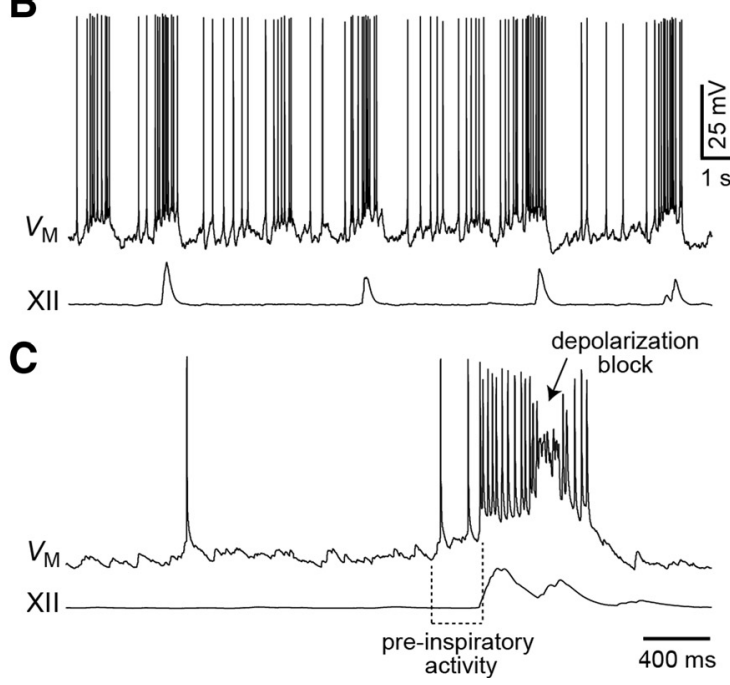

Figure 5. PreBötC Dbx1-derived neurons are respiratory. $\boldsymbol{A}$, EYFP-labeled neurons within the preBötC area of Dbx $1^{\text {CreERT2. }}$ as $\int x / l$. Scale bar, $25 \mu \mathrm{m}$. C, In another Dbx1 ${ }^{+}$neuron from the same slice preparation, the sweep speed is increased to illustrate spiking activity, which are hallmark features of putatively Separate time calibrations are shown for $\boldsymbol{B}$ and $\boldsymbol{C}$.

tile stimulation (11 of 11) (supplemental Video 3, available at www.jneurosci.org as supplemental material). Dbx1 mutant mice do, however, make locomotor movements, respond to stimuli, and show rhythmic heart activity. Furthermore, Dbx1 mutant mice have completely collapsed lungs, indicating the absence of any inspiratory efforts. Dbx1 mutant lung and diaphragm appear otherwise normal (Fig. 7D).

As Dbx1-derived neurons are present throughout the brain, the absence of in vivo behavior may be the consequence of a role for Dbx1 outside the VLM. To address this, we tested whether Dbxl was necessary for inspiratory rhythm in isolated brainstem-spinal cord preparations under conditions known to enhance respiratory activity. In wild-type or Dbx1 $1^{\text {LacZ/+ }}$ E18.5-P0 mice, large-amplitude inspiratory activity was present in all ani-

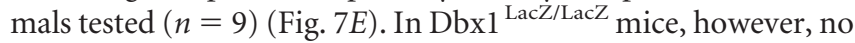
inspiratory activity was present from cervical roots $(n=10)$.

While consistent with the large loss of glutamatergic neurons in the VLM, the absence of rhythmic activity does not necessarily indicate the inability to generate rhythms. We tested whether we could rescue respiratory rhythms in Dbx $1^{\text {LacZ/LacZ }}$ mice by stimulating the remaining network with well established respiratory stimulants: 5-HT and acidosis (Perségol and Viala, 1994; Onimaru et al., 1998; Iizuka, 1999; Peña and Ramirez, 2002; Bodineau et al., 2004; Iizuka, 2004; Kawai et al., 2006; Ptak et al., 2009). Mutant hindbrain preparations exhibited no inspiratory-like output in control aCSF at pH 7.4, nor when exposed to $5 \mu \mathrm{M}$ 5-HT (although tonic cervical nerve discharge increased, as expected (5/5). Decreasing aCSF pH from 7.4 to 7.0 stimulates inspiratory frequency in wild-type animals, but did not induce respiratory-like activity in Dbxl mutant preparations (Fig. 7E). As one further measure, we tested whether a low concentration of SST $\left(10^{-13} \mathrm{M}\right)$ in $\mathrm{pH} 7.0$ aCSF might disinhibit rhythmic cranial root activity as seen with opioids and rhythmic VII nerve activity in rats (Onimaru et al., 2006). However, this combination was without effect in Dbx1 $1^{\text {LacZ/LacZ }}$ mutants (Fig. $7 E$ ). These data indicate that Dbx1 


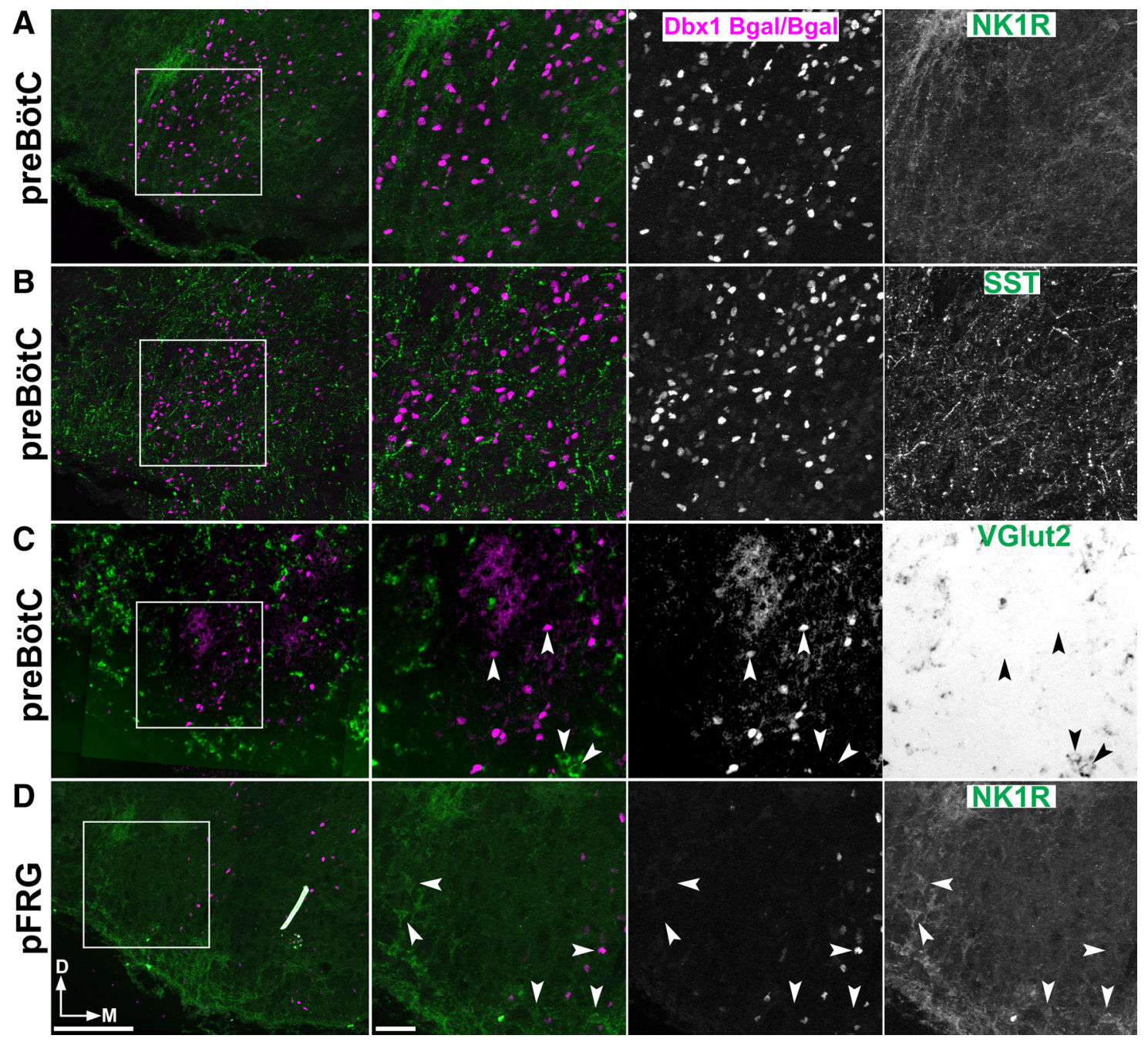

Figure 6. Loss of Dbx1 gene function eliminates preBötC and respiratory glutamatergic neurons. $\boldsymbol{A}-\boldsymbol{C}$, Confocal images showing lack of coexpression of $\beta$-gal (magenta) with NK1R ( $A$, green), SST ( $\boldsymbol{B}$, green), orVGlut2 (C, green, bright-field) in E18.5 Dbx1 ${ }^{\text {LacZ/LacZ }}$ preBötC. $\boldsymbol{D}$, NK1R expression in the pFRG is normal. Note the absence of SST, NK1, and glutamatergic neurons within the preBötC region. Square indicates enlarged images shown to the right. Arrowheads indicate absence of coexpression. Scale bar, $200 \mu \mathrm{m}$. D, Dorsal; M, medial.

loss eliminates the ability to express inspiratory behavior both in vivo and in vitro.

\section{Discussion}

Discovering the origin and identity of the neurons constituting a neural network is key for understanding the neural development and genetic control of behavior (Garcia-Campmany et al., 2010). Here we show that NK1R ${ }^{+} / \mathrm{SST}^{+}$neurons of the preBötC represent a subset of Dbx1-derived neurons. Moreover, Dbx1 specifics nearly all the glutamatergic neurons of the respiratory VLM. Dbx1-derived preBötC neurons are respiratory and Dbx1 gene function is necessary for the formation of respiratory VLM and for the expression of breathing in vivo as well as in vitro. These data suggest that Dbxl is an essential component of the genetic cascade necessary for inspiration, which has important implications for understanding the network underlying breathing. While this paper was in review, a conceptually similar paper also found an essential role for Dbx1 in the formation of the preBötC and for breathing both in vivo and in vitro (Bouvier et al., 2010).

Similar to our results, Bouvier et al. (2010) found that the overwhelming majority of Dbx1 $\beta$-Gal-expressing neurons were glutamatergic and that they made up approximately half of VLM glutamatergic neurons. They did not, however, identify the origin or role of the remaining glutamatergic neurons. We showed, in contrast, that the non-Dbxl-derived glutamatergic neurons within the preBötC region are Lmxlb-derived catecholaminergic neurons important for modulating blood pressure and cardiovascular function and likely not directly involved in breathing. This indicates that all preBötC glutamatergic neurons are Dbxl derived. Our results are consistent with the finding that the elimination of Robo3 from Dbx1-derived neurons is sufficient to eliminate bilateral synchronization of respiratory outputs, as few non-Dbx1derived Robo3-expressing glutamatergic neurons are present (Yuan et al., 2002; Bouvier et al., 2010; Renier et al., 2010).

Within the preBötC, a variety of intracellular criteria, including intrinsic bursting properties and/or ionic conductances, have been used to differentiate putative rhythmogenic from follower neurons. The spectrum of hypotheses regarding the cellular bases for rhythmogenesis has generated vigorous debate (Del Negro et al., 2002; Peña et al., 2004; Ramirez and Garcia, 2007). Our study strongly suggests that all excitatory preBötC respiratory neurons represent variations of a single progenitor population and not distinct developmental populations. This is in sharp contrast to the hypothesized organization of locomotor oscillators where multiple developmentally defined populations play roles in 


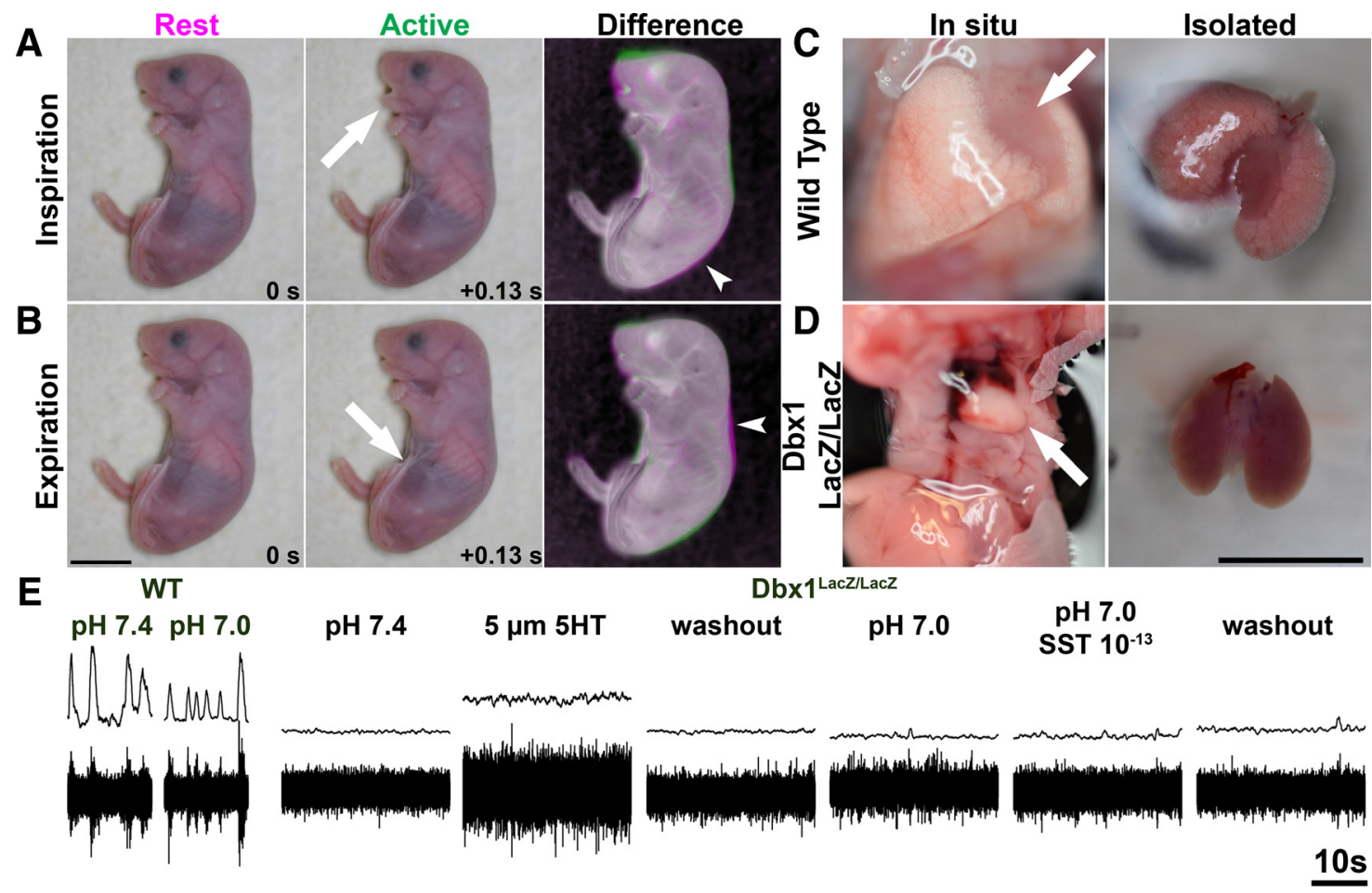

Figure 7. Dbx1 gene function is necessary for breathing in vivo and in vitro. Perinatal mice show two distinct respiratory patterns. Cropped video frames showing the same E18.5 wild-type mouse at rest (left), during an active respiratory movement (middle; $\boldsymbol{A}$, inspiration from supplemental Video $1 ; \boldsymbol{B}$, expiration from supplemental Video 2; both videos available at www.jneurosci.org as supplemental material), and a pseudocolor negative image difference overlay (right). Frames are separated by $0.13 \mathrm{~s}$. Arrows $(\boldsymbol{A}, \boldsymbol{B})$ indicate the region of largest movement. Magenta regions (arrowheads) indicate compression during movement. Green regions indicate expansion during movement. C, Wild-type mice with a respiratory pattern have inflated lungs both in situ and when isolated. $\boldsymbol{D}$, Dbx $1^{\text {LacZ/LacZ }}$ mice have collapsed but normal lungs in situ and when isolated. Scale bar, $1 \mathrm{~cm}$. $\boldsymbol{E}$, Integrated (upper) and raw fictive inspiratory output of C1 from E18.5 WT (left) and Dbx ${ }^{\text {LacZ/LacZ }}$ mice. Wild-type mice show rhythmic respiratory activity and increased response to acidosis (left two traces). Dbx ${ }^{\text {Lacz/lacz }}$ show no respiratory activity in response to $5 \mu \mathrm{M} 5$-HT, acidosis, or acidosis and SST $\left(10^{-13} \mathrm{M}\right)$. Note increases in baseline motor activity after 5 -HT. Calibration: $10 \mathrm{~s}$.

rhythm and pattern generation (Kozlov et al., 2009; GarciaCampmany et al., 2010).

Phase of firing during the respiratory cycle has also been proposed to identify distinct functional subclasses of preBötC neurons (Smith et al., 1990; Rekling et al., 1996a; Koshiya and Smith, 1999; Thoby-Brisson et al., 2000; Thoby-Brisson and Ramirez, 2001; Peña et al., 2004; Ramirez and Viemari, 2005; Koizumi et al., 2008; Rubin et al., 2009). In E15.5 rhythmically active slices, Bouvier et al. (2010) found that nearly $85 \%$ of Dbx1-derived neurons were rhythmically active and in phase with inspiratory output as measured by calcium imaging, and that $70 \%$ were rhythmically active and received phasic inspiratory depolarization with whole-cell recordings. We extend these results and find that an early-I firing phenotype in postnatal rhythmic slices is a characteristic property of nearly $95 \%$ of postnatal Dbx1-derived glutamatergic neurons. This is in contrast to the firing pattern of genetically identified inspiratory glycinergic neurons within the preBötC, which begin their inspiratory-related firing nearly in sync with motor output (Morgado-Valle et al., 2010). What role intrinsic conductances and/or network synaptic process play in the early-I phenotype is still incompletely understood (Rubin et al., 2009). Nevertheless, these data suggest that in the absence of recordings from genetically labeled preBötC glutamatergic neurons, an early-I firing pattern represents a reasonable approximation for identifying excitatory neurons. Further, they suggest that transmitter identity can be inferred from the rhythmic inspiratory firing patterns of neurons in the literature (Thoby-Brisson and Ramirez, 2001).

The respiratory portion of the VLM contains anatomically and functionally distinct subdivisions that contain both excita- tory and inhibitory interneurons in addition to the preBötC (Schreihofer et al., 1999; Alheid and McCrimmon, 2008). Bouvier et al. (2010) focused exclusively on the development of Dbx1-derived neurons of the preBötC. Our finding that Dbx1derived neurons extend throughout the VLM and represent nearly all VLM non-catecholaminergic, glutamatergic neurons suggests that excitatory neurons outside the preBötC play a role in shaping respiratory output. This is further reinforced by the heterogeneous pattern of NK1R and SST2aR expression within the VLM that may explain the ability of Substance P to promote sigh activity in vitro by acting on rostral brainstem regions (Lieske et al., 2000; Doi and Ramirez, 2008; Ruangkittisakul et al., 2008). The recent finding that the introduction of a fragment of the SST promoter into preBötC neurons in adult rat drives expression of GFP in a larger percentage of preBötC neurons than normally express SST is consistent with a developmentally shared origin of all preBötC excitatory neurons (Tan et al., 2010). We also find that the VLM contains a subset of Dbx1-derived glia, whose specific role in respiratory modulation is still incompletely understood (Gourine et al., 2010).

Whether the specific peptide or peptide receptor expression within individual VLM neurons identifies unique functional populations or merely components of a larger distributed network is unknown. We hypothesize that preBötC core neurons represent a highly connected, and thus specialized, subset for communicating respiratory oscillations to the larger Dbx1derived respiratory population and that their elimination affects breathing by disrupting the propagation of excitatory neurotransmission in the respiratory network (Del Negro et al., 2002; Rubin et al., 2009). The extent to which Dbx1-derived or other 
glutamatergic neurons outside the preBötC core can show plasticity after preBötC lesion remains to be determined (Gray et al., 2001; McKay et al., 2005; McKay and Feldman, 2008). The finding that Dbx1-derived, glutamatergic neurons extend throughout the VLM, however, suggests that Dbx1-derived neurons outside the preBötC may be capable of aspects of respiratory rhythm generation given the maintenance of sufficient connectivity (Forster et al., 2010; Neumueller et al., 2010).

Dbx1 mutant mice do not generate either inspiratory or expiratory output in vivo, nor do they generate inspiratory output in vitro. The data suggest that the loss of inspiratory activity in Dbx1 mutants is a direct consequence of the loss of VLM glutamatergic neurons and that remaining brainstem populations are insufficient to recover respiratory output. We further hypothesize the inspiratory rhythm is generated by VLM Dbx1-derived neurons, including the preBötC, as Dbx1-derived neurons meet many of the criteria for respiratory rhythm generation (Feldman and Del Negro, 2006). It should be pointed out, however, that Dbx1derived neurons in the spinal cord have been shown to directly drive motoneurons (Lanuza et al., 2004). It is possible that the absence of respiratory behavior may be at least in part the consequence of the inability of non-Dbx1-derived neurons outside the VLM to propagate their signal to motoneurons (Koizumi et al., 2008). While the loss of inspiratory activity might be expected by the absence of VLM glutamatergic neurons, expiratory activity has been proposed to be generated by Atoh1- and/or Phox2bexpressing populations. It remains to be determined whether Dbx1-derived populations within the VLM or elsewhere in the CNS are necessary for the expression of independent expiratory activity in vivo or in vitro.

In summary, we provide a genetic description for a population of neurons necessary for a fundamental homeostatic behavior, breathing. These data suggest that the network underlying the breathing rhythm is genetically encoded and may represent an ideal model system for examining the functional, developmental, and evolutionary origins of behavior. We propose that breathing behavior is generated by a genetically definable population and that all the properties that comprise this quintessential mammalian $\mathrm{CPG}$, including the rhythmogenic, synaptic, and intrinsic membrane properties of the constituent neurons, can be generated from a single developmental precursor population. These data may provide a genetic framework for addressing the evolution of neural circuits as well as for understanding disorders of breathing with a central etiology, such as apnea of prematurity, sudden infant death syndrome, central sleep apnea, and respiratory failure in neurodegenerative disorders such as multiple system atrophy or Perry syndrome (Feldman and Del Negro, 2006).

\section{References}

Alheid GF, McCrimmon DR (2008) The chemical neuroanatomy of breathing. Respir Physiol Neurobiol 164:3-11.

Ballanyi K, Ruangkittisakul A, Onimaru H (2009) Opioids prolong and anoxia shortens delay between onset of preinspiratory (pFRG) and inspiratory (preBotC) network bursting in newborn rat brainstems. Pflugers Arch 458:571-587.

Bielle F, Griveau A, Narboux-Nême N, Vigneau S, Sigrist M, Arber S, Wassef M, Pierani A (2005) Multiple origins of Cajal-Retzius cells at the borders of the developing pallium. Nat Neurosci 8:1002-1012.

Blanchi B, Kelly LM, Viemari JC, Lafon I, Burnet H, Bévengut M, Tillmanns S, Daniel L, Graf T, Hilaire G, Sieweke MH (2003) MafB deficiency causes defective respiratory rhythmogenesis and fatal central apnea at birth. Nat Neurosci 6:1091-1100.

Bodineau L, Cayetanot F, Marlot D, Collin T, Gros F, Frugière A (2004) Endogenous 5-HT(1/2) systems and the newborn rat respiratory control.
A comparative in vivo and in vitro study. Respir Physiol Neurobiol 141:47-57.

Bouvier J, Thoby-Brisson M, Renier N, Dubreuil V, Ericson J, Champagnat J, Pierani A, Chédotal A, Fortin G (2010) Hindbrain interneurons and axon guidance signaling critical for breathing. Nat Neurosci 13:10661074.

Burke PG, Abbott SB, McMullan S, Goodchild AK, Pilowsky PM (2010) Somatostatin selectively ablates post-inspiratory activity after injection into the Botzinger complex. Neuroscience 167:528-539.

Buscail L, Delesque N, Estève JP, Saint-Laurent N, Prats H, Clerc P, Robberecht P, Bell GI, Liebow C, Schally AV, Vaysse N, Susini C (1994) Stimulation of tyrosine phosphatase and inhibition of cell proliferation by somatostatin analogues: mediation by human somatostatin receptor subtypes SSTR1 and SSTR2. Proc Natl Acad Sci U S A 91:2315-2319.

Butera RJ Jr, Rinzel J, Smith JC (1999) Models of respiratory rhythm generation in the pre-Botzinger complex. I. Bursting pacemaker neurons. J Neurophysiol 82:382-397.

Caubit X, Thoby-Brisson M, Voituron N, Filippi P, Bévengut M, Faralli H, Zanella S, Fortin G, Hilaire G, Fasano L (2010) Teashirt 3 regulates development of neurons involved in both respiratory rhythm and airflow control. J Neurosci 30:9465-9476.

Chattopadhyaya B, Di Cristo G, Higashiyama H, Knott GW, Kuhlman SJ, Welker E, Huang ZJ (2004) Experience and activity-dependent maturation of perisomatic GABAergic innervation in primary visual cortex during a postnatal critical period. J Neurosci 24:9598-9611.

Del Negro CA, Johnson SM, Butera RJ, Smith JC (2001) Models of respiratory rhythm generation in the pre-Botzinger complex. III. Experimental tests of model predictions. J Neurophysiol 86:59-74.

Del Negro CA, Morgado-Valle C, Feldman JL (2002) Respiratory rhythm: an emergent network property? Neuron 34:821-830.

Del Negro CA, Morgado-Valle C, Hayes JA, Mackay DD, Pace RW, Crowder EA, Feldman JL (2005) Sodium and calcium current-mediated pacemaker neurons and respiratory rhythm generation. J Neurosci 25:446-453.

Doi A, Ramirez JM (2008) Neuromodulation and the orchestration of the respiratory rhythm. Respir Physiol Neurobiol 164:96-104.

Doi A, Ramirez JM (2010) State-dependent interactions between excitatory neuromodulators in the neuronal control of breathing. J Neurosci 30:8251-8262.

Feldman JL, Del Negro CA (2006) Looking for inspiration: new perspectives on respiratory rhythm. Nat Rev Neurosci 7:232-242.

Feldman JL, Smith JC (1989) Cellular mechanisms underlying modulation of breathing pattern in mammals. Ann NY Acad Sci 563:114-130.

Feldman JL, Smith JC, Ellenberger HH, Connelly CA, Liu GS, Greer JJ, Lindsay AD, Otto MR (1990) Neurogenesis of respiratory rhythm and pattern: emerging concepts. Am J Physiol 259:R879-R886.

Fogarty M, Richardson WD, Kessaris N (2005) A subset of oligodendrocytes generated from radial glia in the dorsal spinal cord. Development 132:1951-1959.

Forster HV, Krause KL, Kiner T, Neumueller SE, Bonis JM, Qian B, Pan LG (2010) Plasticity of respiratory rhythm-generating mechanisms in adult goats. Adv Exp Med Biol 669:151-155.

Garcia-Campmany L, Stam FJ, Goulding M (2010) From circuits to behaviour: motor networks in vertebrates. Curr Opin Neurobiol 20:116-125.

Gong S, Zheng C, Doughty ML, Losos K, Didkovsky N, Schambra UB, Nowak NJ, Joyner A, Leblanc G, Hatten ME, Heintz N (2003) A gene expression atlas of the central nervous system based on bacterial artificial chromosomes. Nature 425:917-925.

Gourine AV, Kasymov V, Marina N, Tang F, Figueiredo MF, Lane S, Teschemacher AG, Spyer KM, Deisseroth K, Kasparov S (2010) Astrocytes control breathing through $\mathrm{pH}$-dependent release of ATP. Science 329:571-575.

Gray PA (2008) Transcription factors and the genetic organization of brain stem respiratory neurons. J Appl Physiol 104:1513-1521.

Gray PA, Rekling JC, Bocchiaro CM, Feldman JL (1999) Modulation of respiratory frequency by peptidergic input to rhythmogenic neurons in the preBotzinger complex. Science 286:1566-1568.

Gray PA, Janczewski WA, Mellen N, McCrimmon DR, Feldman JL (2001) Normal breathing requires preBotzinger complex neurokinin-1 receptorexpressing neurons. Nat Neurosci 4:927-930.

Gray PA, Fu H, Luo P, Zhao Q, Yu J, Ferrari A, Tenzen T, Yuk DI, Tsung EF, Cai Z, Alberta JA, Cheng LP, Liu Y, Stenman JM, Valerius MT, Billings N, 
Kim HA, Greenberg ME, McMahon AP, Rowitch DH, et al. (2004) Mouse brain organization revealed through direct genome-scale TF expression analysis. Science 306:2255-2257.

Guyenet PG, Wang H (2001) Pre-Botzinger neurons with preinspiratory discharges "in vivo" express NK1 receptors in the rat. J Neurophysiol 86:438-446.

Hayes JA, Del Negro CA (2007) Neurokinin receptor-expressing preBotzinger complex neurons in neonatal mice studied in vitro. J Neurophysiol 97:4215-4224.

Hirata T, Li P, Lanuza GM, Cocas LA, Huntsman MM, Corbin JG (2009) Identification of distinct telencephalic progenitor pools for neuronal diversity in the amygdala. Nat Neurosci 12:141-149.

Iizuka M (1999) Intercostal expiratory activity in an in vitro brainstemspinal cord-rib preparation from the neonatal rat. J Physiol 520:293-302.

Iizuka M (2004) Rostrocaudal distribution of spinal respiratory motor activity in an in vitro neonatal rat preparation. Neurosci Res 50:263-269.

Janczewski WA, Feldman JL (2006a) Distinct rhythm generators for inspiration and expiration in the juvenile rat. J Physiol 570:407-420.

Janczewski WA, Feldman JL (2006b) Novel data supporting the two respiratory rhythm oscillator hypothesis. Focus on "respiration-related rhythmic activity in the rostral medulla of newborn rats." J Neurophysiol 96:1-2.

Kawai A, Onimaru H, Homma I (2006) Mechanisms of CO2/H+ chemoreception by respiratory rhythm generator neurons in the medulla from newborn rats in vitro. J Physiol 572:525-537.

Koizumi H, Wilson CG, Wong S, Yamanishi T, Koshiya N, Smith JC (2008) Functional imaging, spatial reconstruction, and biophysical analysis of a respiratory motor circuit isolated in vitro. J Neurosci 28:2353-2365.

Koshiya N, Smith JC (1999) Neuronal pacemaker for breathing visualized in vitro. Nature 400:360-363.

Kozlov A, Huss M, Lansner A, Kotaleski JH, Grillner S (2009) Simple cellular and network control principles govern complex patterns of motor behavior. Proc Natl Acad Sci U S A 106:20027-20032.

Lanuza GM, Gosgnach S, Pierani A, Jessell TM, Goulding M (2004) Genetic identification of spinal interneurons that coordinate left-right locomotor activity necessary for walking movements. Neuron 42:375-386.

Legallois M (1813) Experiments on the principle of life. Philadelphia: Thomas.

Lieske SP, Thoby-Brisson M, Telgkamp P, Ramirez JM (2000) Reconfiguration of the neural network controlling multiple breathing patterns: eupnea, sighs and gasps [see comment]. Nat Neurosci 3:600-607.

Liu B, Liu Z, Chen T, Li H, Qiang B, Yuan J, Peng X, Qiu M (2007) Selective expression of Bhlhb5 in subsets of early-born interneurons and late-born association neurons in the spinal cord. Dev Dyn 236:829-835.

Llona I, Eugenín J (2005) Central actions of somatostatin in the generation and control of breathing. Biol Res 38:347-352.

McKay LC, Feldman JL (2008) Unilateral ablation of pre-Botzinger complex disrupts breathing during sleep but not wakefulness. Am J Respir Crit Care Med 178:89-95.

McKay LC, Janczewski WA, Feldman JL (2005) Sleep-disordered breathing after targeted ablation of preBotzinger complex neurons. Nat Neurosci $8: 1142-1144$

Mellen NM, Janczewski WA, Bocchiaro CM, Feldman JL (2003) Opioidinduced quantal slowing reveals dual networks for respiratory rhythm generation. Neuron 37:821-826.

Morgado-Valle C, Baca SM, Feldman JL (2010) Glycinergic pacemaker neurons in preBotzinger complex of neonatal mouse. J Neurosci 30:3634-3639.

Nattie E, Li A (2006) Neurokinin-1 receptor-expressing neurons in the ventral medulla are essential for normal central and peripheral chemoreception in the conscious rat. J Appl Physiol 101:1596-1606.

Nattie EE, Li A (2002) Substance P-saporin lesion of neurons with NK1 receptors in one chemoreceptor site in rats decreases ventilation and chemosensitivity. J Physiol 544:603-616.

Neumueller S, Hodges MR, Krause K, Marshall B, Bonis J, Qian B, Pan LG, Forster HV (2010) Anatomic changes in multiple brainstem nuclei after incremental, near-complete neurotoxic destruction of the pre-Botzinger complex in adult goats. Respir Physiol Neurobiol. Advance online publication. Retrieved June 16, 2010. doi:10.1016/j.resp.2010.06.010.

Onimaru H, Shamoto A, Homma I (1998) Modulation of respiratory rhythm by 5-HT in the brainstem-spinal cord preparation from newborn rat. Pflugers Arch 435:485-494.
Onimaru H, Kumagawa Y, Homma I (2006) Respiration-related rhythmic activity in the rostral medulla of newborn rats. J Neurophysiol 96:55-61.

Pagliardini S, Ren J, Greer JJ (2003) Ontogeny of the pre-Botzinger complex in perinatal rats. J Neurosci 23:9575-9584.

Pagliardini S, Ren J, Gray PA, Vandunk C, Gross M, Goulding M, Greer JJ (2008) Central respiratory rhythmogenesis is abnormal in lbx1- deficient mice. J Neurosci 28:11030-11041.

Peña F, Ramirez JM (2002) Endogenous activation of serotonin-2A receptors is required for respiratory rhythm generation in vitro. J Neurosci 22:11055-11064.

Peña F, Parkis MA, Tryba AK, Ramirez JM (2004) Differential contribution of pacemaker properties to the generation of respiratory rhythms during normoxia and hypoxia. Neuron 43:105-117.

Perségol L, Viala D (1994) Characteristics of slow bursting activities recorded in cervical ventral roots in the in vitro brainstem-spinal cord preparation of the neonatal rat. Somatosens Mot Res 11:57-64.

Pierani A, Moran-Rivard L, Sunshine MJ, Littman DR, Goulding M, Jessell TM (2001) Control of interneuron fate in the developing spinal cord by the progenitor homeodomain protein Dbx1. Neuron 29:367-384.

Pressman CL, Chen H, Johnson RL (2000) LMX1B, a LIM homeodomain class transcription factor, is necessary for normal development of multiple tissues in the anterior segment of the murine eye. Genesis 26:15-25.

Ptak K, Yamanishi T, Aungst J, Milescu LS, Zhang R, Richerson GB, Smith JC (2009) Raphe neurons stimulate respiratory circuit activity by multiple mechanisms via endogenously released serotonin and substance P. J Neurosci 29:3720-3737.

Qian Y, Shirasawa S, Chen CL, Cheng L, Ma Q (2002) Proper development of relay somatic sensory neurons and D2/D4 interneurons requires homeobox genes Rnx/Tlx-3 and Tlx-1. Genes Dev 16:1220-1233.

Ramirez JM, Garcia A 3rd (2007) Point: medullary pacemaker neurons are essential for both eupnea and gasping in mammals. J Appl Physiol 103: 717-718; discussion 722 .

Ramirez JM, Viemari JC (2005) Determinants of inspiratory activity. Respir Physiol Neurobiol 147:145-157.

Rekling JC, Champagnat J, Denavit-Saubié M (1996a) Electroresponsive properties and membrane potential trajectories of three types of inspiratory neurons in the newborn mouse brain stem in vitro. J Neurophysiol 75:795-810.

Rekling JC, Champagnat J, Denavit-Saubié M (1996b) Thyrotropinreleasing hormone (TRH) depolarizes a subset of inspiratory neurons in the newborn mouse brain stem in vitro. J Neurophysiol 75:811-819.

Renier N, Schonewille M, Giraudet F, Badura A, Tessier-Lavigne M, Avan P, De Zeeuw CI, Chédotal A (2010) Genetic dissection of the function of hindbrain axonal commissures. PLoS Biol 8:e1000325.

Rhee JW, Arata A, Selleri L, Jacobs Y, Arata S, Onimaru H, Cleary ML (2004) $\mathrm{Pbx} 3$ deficiency results in central hypoventilation. Am J Pathol 165:1343-1350.

Richter DW (1982) Generation and maintenance of the respiratory rhythm. J Exp Biol 100:93-107.

Rose MF, Ren J, Ahmad KA, Chao HT, Klisch TJ, Flora A, Greer JJ, Zoghbi HY (2009) Math1 is essential for the development of hindbrain neurons critical for perinatal breathing. Neuron 64:341-354

Ruangkittisakul A, Schwarzacher SW, Secchia L, Poon BY, Ma Y, Funk GD, Ballanyi K (2006) High sensitivity to neuromodulator-activated signaling pathways at physiological $\left[\mathrm{K}^{+}\right]$of confocally imaged respiratory center neurons in on-line-calibrated newborn rat brainstem slices. J Neurosci 26:11870-11880.

Ruangkittisakul A, Schwarzacher SW, Secchia L, Ma Y, Bobocea N, Poon BY, Funk GD, Ballanyi K (2008) Generation of eupnea and sighs by a spatiochemically organized inspiratory network. J Neurosci 28:2447-2458.

Rubin JE, Hayes JA, Mendenhall JL, Del Negro CA (2009) Calciumactivated nonspecific cation current and synaptic depression promote network-dependent burst oscillations. Proc Natl Acad Sci U S A 106: 2939-2944.

Schindler M, Holloway S, Hathway G, Woolf CJ, Humphrey PP, Emson PC (1998) Identification of somatostatin sst2(a) receptor expressing neurones in central regions involved in nociception. Brain Res 798:25-35.

Schreihofer AM, Stornetta RL, Guyenet PG (1999) Evidence for glycinergic respiratory neurons: Bötzinger neurons express mRNA for glycinergic transporter 2. J Comp Neurol 407:583-597.

Schulz S, Händel M, Schreff M, Schmidt H, Höllt V (2000) Localization of 
five somatostatin receptors in the rat central nervous system using subtype-specific antibodies. J Physiol Paris 94:259-264.

Smith JC, Greer JJ, Liu GS, Feldman JL (1990) Neural mechanisms generating respiratory pattern in mammalian brain stem-spinal cord in vitro. I. Spatiotemporal patterns of motor and medullary neuron activity. J Neurophysiol 64:1149-1169.

Smith JC, Ellenberger HH, Ballanyi K, Richter DW, Feldman JL (1991) PreBötzinger complex: a brainstem region that may generate respiratory rhythm in mammals. Science 254:726-729.

Smith JC, Abdala AP, Koizumi H, Rybak IA, Paton JF (2007) Spatial and functional architecture of the mammalian brain stem respiratory network: a hierarchy of three oscillatory mechanisms. J Neurophysiol 98:3370-3387.

Srinivas S, Watanabe T, Lin CS, William CM, Tanabe Y, Jessell TM, Costantini F (2001) Cre reporter strains produced by targeted insertion of EYFP and ECFP into the ROSA26 locus. BMC Dev Biol 1:4.

Stornetta RL, Sevigny CP, Guyenet PG (2002) Vesicular glutamate transporter DNPI/VGLUT2 mRNA is present in $\mathrm{C} 1$ and several other groups of brainstem catecholaminergic neurons. J Comp Neurol 444:191-206.

Stornetta RL, Rosin DL, Wang H, Sevigny CP, Weston MC, Guyenet PG (2003) A group of glutamatergic interneurons expressing high levels of both neurokinin- 1 receptors and somatostatin identifies the region of the pre-Botzinger complex. J Comp Neurol 455:499-512.

Stornetta RL, Moreira TS, Takakura AC, Kang BJ, Chang DA, West GH, Brunet JF, Mulkey DK, Bayliss DA, Guyenet PG (2006) Expression of Phox $2 \mathrm{~b}$ by brainstem neurons involved in chemosensory integration in the adult rat. J Neurosci 26:10305-10314.

Takakura AC, Moreira TS, Stornetta RL, West GH, Gwilt JM, Guyenet PG (2008) Selective lesion of retrotrapezoid Phox2b-expressing neurons raises the apnoeic threshold in rats. J Physiol 586:2975-2991.

Takeda S, Eriksson LI, Yamamoto Y, Joensen H, Onimaru H, Lindahl SG (2001) Opioid action on respiratory neuron activity of the isolated respiratory network in newborn rats. Anesthesiology 95:740-749.

Tan W, Janczewski WA, Yang P, Shao XM, Callaway EM, Feldman JL (2008) Silencing preBotzinger complex somatostatin-expressing neurons induces persistent apnea in awake rat. Nat Neurosci 11:538-540.
Tan W, Pagliardini S, Yang P, Janczewski WA, Feldman JL (2010) Projections of preBotzinger complex neurons in adult rats. J Comp Neurol 518:1862-1878.

Thoby-Brisson M, Ramirez JM (2001) Identification of two types of inspiratory pacemaker neurons in the isolated respiratory neural network of mice. J Neurophysiol 86:104-112.

Thoby-Brisson M, Telgkamp P, Ramirez JM (2000) The role of the hyperpolarization-activated current in modulating rhythmic activity in the isolated respiratory network of mice. J Neurosci 20:2994-3005.

Thoby-Brisson M, Trinh JB, Champagnat J, Fortin G (2005) Emergence of the pre-Botzinger respiratory rhythm generator in the mouse embryo. J Neurosci 25:4307-4318.

Thoby-Brisson M, Karlén M, Wu N, Charnay P, Champagnat J, Fortin G (2009) Genetic identification of an embryonic parafacial oscillator coupling to the preBotzinger complex. Nat Neurosci 12:1028-1035.

Wallén-Mackenzie A, Gezelius H, Thoby-Brisson M, Nygård A, Enjin A, Fujiyama F, Fortin G, Kullander K (2006) Vesicular glutamate transporter 2 is required for central respiratory rhythm generation but not for locomotor central pattern generation. J Neurosci 26:12294-12307.

Wang H, Weston MC, McQuiston TJ, Stornetta RL, Guyenet PG (2003) Neurokinin-1 receptor-expressing cells regulate depressor region of rat ventrolateral medulla. Am J Physiol Heart Circ Physiol 285:H2757-H2769.

Winter SM, Hirrlinger J, Kirchhoff F, Hülsmann S (2007) Transgenic expression of fluorescent proteins in respiratory neurons. Respir Physiol Neurobiol 159:108-114.

Winter SM, Fresemann J, Schnell C, Oku Y, Hirrlinger J, Hülsmann S (2010) Glycinergic interneurons in the respiratory network of the rhythmic slice preparation. Adv Exp Med Biol 669:97-100.

Yuan SS, Yeh YT, Lee EY (2002) Pax-2 interacts with RB and reverses its repression on the promoter of Rig-1, a Robo member. Biochem Biophys Res Commun 296:1019-1025.

Zagoraiou L, Akay T, Martin JF, Brownstone RM, Jessell TM, Miles GB (2009) A cluster of cholinergic premotor interneurons modulates mouse locomotor activity. Neuron 64:645-662. 